Article

\title{
Generalizations of the Nonlinear Henry Inequality and the Leray-Schauder Type Fixed Point Theorem with Applications to Fractional Differential Inclusions
}

\author{
Zouaoui Hamlat $^{1}$, John R. Graef ${ }^{2, *}$ and Abdelghani Ouahab ${ }^{1}$ \\ 1 Laboratory of Mathematics, University of Djillali Liabès, P.O. Box 89, Sidi-Bel-Abbès 22000, Algeria; \\ hamlet.hamlat@gmail.com (Z.H.); agh_ouahab@yahoo.fr (A.O.) \\ 2 Department of Mathematics, University of Tennessee at Chattanooga, Chattanooga, TN 37403, USA \\ * Correspondence: John-Graef@utc.edu
}

Citation: Hamlat, Z.; Graef, J.R.; Ouahab, A. Generalizations of the Nonlinear Henry Inequality and the Leray-Schauder Type Fixed Point Theorem with Applications to Fractional Differential Inclusions. Fractal Fract. 2021, 5, 173. https:// doi.org/10.3390/fractalfract5040173

Academic Editor: Ravi P. Agarwal

Received: 2 July 2021

Accepted: 1 October 2021

Published: 18 October 2021

Publisher's Note: MDPI stays neutral with regard to jurisdictional claims in published maps and institutional affiliations.

Copyright: (c) 2021 by the authors. Licensee MDPI, Basel, Switzerland. This article is an open access article distributed under the terms and conditions of the Creative Commons Attribution (CC BY) license (https:/ / creativecommons.org/licenses/by/ $4.0 /)$.

\begin{abstract}
The authors give some singular versions of the Gronwall-Bihari-Henry inequalities. They also establish a multivalued version of the Leray-Schauder alternative in strictly star-shaped sets. Based on these new fractional inequalities and fixed point theorem, they study an initial value problem for fractional differential inclusions with delay.
\end{abstract}

Keywords: fractional differential equations; bihari inequality; fractional integral; fractional derivative; fixed point; Leray-Schauder condition; interior condition; star-shape set

MSC: 26A33; 26A42; 34A60; 54H25

\section{Introduction}

It is well-known that inequalities, such as the Gronwall-Bellman-Bihari-Henry inequality, play an important role in the study of existence, uniqueness, boundedness, stability, and other qualitative properties of solutions of differential equations, integral equations, and differential inclusions (see, for example, [1-7]). For nonlinear integral inequalities, perhaps one of the most important contributions was made by Bihari [8] for

$$
v(t) \leq k+\int_{0}^{t} f(s) \psi(v(s)) d s, \quad k \in \mathbb{R}_{+} .
$$

This integral inequality was generalized by many authors. For example, Agarwal et al. [9] replaced $k, t, f$, and $\psi$ with the functions $a(t),\left(b_{i}(t)\right)_{i=1}^{p},\left(f_{i}\right)_{i=1}^{p}$, and $\left(\psi_{i}\right)_{i=1}^{p}$, respectively, and investigated the retarded Gronwall type inequality

$$
v(t) \leq a(t)+\sum_{i=1}^{p} \int_{b_{i}(0)}^{b_{i}(t)} f_{i}(s) \psi_{i}(v(s)) d s, b(t) \leq t, 0 \leq t \leq T .
$$

In recent years, ordinary and partial differential equations of fractional order have been investigated more in the literature due to their applicability to many problems in engineering and other scientific disciplines; see [10-15] and the references therein for recent work. The question of the existence of solutions and other mathematical aspects of fractional differential equations and inclusions have been extensively studied and have attracted much attention; many important contributions have been obtained so far (see the monographs [16-18] as well as papers listed in the references below).

Linear and nonlinear integral inequalities with singular kernels have received considerable attention in the literature since 1981 when Henry [3] established the following result: 
If $w$ is a non-negative locally integrable function, $a \geq 0$ is a constant, $0<\gamma<1$, and

$$
v(t) \leq w(t)+a \int_{0}^{t} \frac{v(s)}{(t-s)^{\gamma}} d s,
$$

then there exists a constant $K=K(\gamma)$ such that

$$
v(t) \leq w(t)+K a \int_{0}^{t} \frac{w(s)}{(t-s)^{\gamma}} d s, \text { for } t \in[0, b] .
$$

Henry's result has been extended to more general linear integral inequalities by many authors such as in [3,19-23]. All these results are proved by an iteration argument and the results expressed as integrals with singular kernels often defined by power series of very complicated forms that are sometimes not very convenient for applications.

In $[24,25]$, Medved studied the nonlinear integral inequality of Henry type

$$
v(t) \leq w(t)+a \int_{0}^{t}(t-s)^{\alpha-1} \psi(v(s)) d s
$$

where $\alpha<1$ and $\psi$ is a positive nondecreasing function.

The aim of the present paper is to establish some new and useful nonlinear generalizations of the integral inequality given in [3]; we also generalize the singular inequality of Agarwal et al. [9]. In our proofs, we make use of the Young and Hölder inequalities combined with a classical Bihari type inequality to obtain our results.

It is also our goal to establish a new multivalued version of the Leray-Schauder fixed point theorem. In order to accomplish this, we first recall some notions from multivariate analysis in Section 3. In Section 4, we prove our Leray-Schauder type fixed point theorem for multivalued mappings. Then, in Section 5, we use the fractional inequalities derived in Section 2 and apply our fixed point theorem from Section 4 to an initial value problem for a fractional delay differential inclusion in star shaped sets.

\section{A Nonlinear Integral Inequality}

In this section, we wish to establish some nonlinear integral inequalities that can be used in the analysis of fractional differential equations and inclusions. The proofs are based on Young's and Hölder's inequalities.

Theorem 1. Let $0<\alpha<1, q>\frac{1}{\alpha}$, and $k_{0}, k_{1}, k_{2}>0$. For $i=1,2, \ldots, n$, let $f_{i}, \lambda_{i}, \gamma_{i}$, and $g$ be non-negative functions that are locally integrable on $I=[0, T]$, and let $\psi_{i}:[0, \infty) \rightarrow[0, \infty)$ be nondecreasing continuous functions. If $u(t)$ is a non-negative continuous function on I satisfying

$$
u(t) \leq k_{0}+\sum_{i=1}^{n} k_{1} \int_{0}^{t}(t-s)^{\alpha-1} g(s) \gamma_{i}(s) u(s) d s+\sum_{i=1}^{n} k_{2} \int_{0}^{t}(t-s)^{\alpha-1} f_{i}(s) \lambda_{i}(s) \psi_{i}(u(s)) d s,
$$

then

$$
u(t) \leq \Psi^{-1}\left(\Psi(\widetilde{k})+\int_{0}^{t} m(s) d s\right)
$$

where $\frac{1}{p}+\frac{1}{q}=1$,

$$
\begin{gathered}
m(t)=\max \left(\sum_{i=1}^{n} \frac{1}{q}\left(\gamma_{i}(t) g(t)\right)^{q}, \sum_{i=1}^{n} \frac{1}{q}\left(\lambda_{i}(t) f_{i}(t)\right)^{q}\right), \widetilde{k}=k_{0}+\frac{\left(k_{1}^{p}+k_{2}^{p}\right) n T^{p(\alpha-1)+1}}{p(p(\alpha-1)+1)}, \\
\Psi(z)=\int_{k_{0}}^{z} \frac{d y}{y^{q}+\widetilde{\psi}(y)}, \widetilde{\psi}(y)=\max \left\{\psi_{i}^{q}(y): i=1,2, \ldots, n\right\},
\end{gathered}
$$


$\Psi^{-1}$ is the inverse function of $\Psi, \operatorname{Dom}\left(\Psi^{-1}\right)$ is the domain of $\Psi^{-1}$, and for every $t \in[0, T]$,

$$
\Psi(\widetilde{k})+\int_{0}^{t} m(s) d s \in \operatorname{Dom}\left(\Psi^{-1}\right) .
$$

Proof. By Young's inequality, we see that

$$
k_{1}(t-s)^{\alpha-1} g(s) \gamma_{i}(s) u(s) \leq \frac{k_{1}^{p}}{p}(t-s)^{p(\alpha-1)}+\frac{1}{q}\left(\gamma_{i}(s) g(s) u(s)\right)^{q},
$$

and

$$
k_{2}(t-s)^{\alpha-1} f_{i}(s) \lambda_{i}(s) \psi_{i}(u(s)) \leq \frac{k_{2}^{p}}{p}(t-s)^{p(\alpha-1)}+\frac{1}{q}\left(\lambda_{i}(s) f_{i}(s)\right)^{q}\left(\psi_{i}(u(s))\right)^{q} .
$$

Then,

$$
\begin{aligned}
& u(t) \leq k_{0}+\sum_{i=1}^{n}\left(\frac{k_{1}^{p}}{p} \int_{0}^{t}(t-s)^{p(\alpha-1)}+\frac{1}{q} \int_{0}^{t}\left(\gamma_{i}(s) g(s) u(s)\right)^{q} d s\right) \\
& +\sum_{i=1}^{n}\left(\frac{k_{2}^{p}}{p} \int_{0}^{t}(t-s)^{p(\alpha-1)}+\frac{1}{q} \int_{0}^{t}\left(\lambda_{i}(s) f_{i}(s)\right)^{q}\left(\psi_{i}(u(s))\right)^{q} d s\right) \\
& \leq k_{0}+\frac{\left(k_{1}^{p}+k_{2}^{p}\right) n T^{p(\alpha-1)+1}}{p(p(\alpha-1)+1)}+\sum_{i=1}^{n} \frac{1}{q} \int_{0}^{t}\left(\gamma_{i}(s) g(s) u(s)\right)^{q} d s \\
& +\sum_{i=1}^{n} \frac{1}{q} \int_{0}^{t}\left(\lambda_{i}(s) f_{i}(s)\right)^{q}\left(\psi_{i}(u(s))\right)^{q} d s \\
& \leq \widetilde{k}+\sum_{i=1}^{n} \frac{1}{q} \int_{0}^{t}\left(\gamma_{i}(s) g(s) u(s)\right)^{q} d s+\sum_{i=1}^{n} \frac{1}{q} \int_{0}^{t}\left(\lambda_{i}(s) f_{i}(s)\right)^{q}\left(\psi_{i}(u(s))\right)^{q} d s,
\end{aligned}
$$

where

$$
\widetilde{k}=k_{0}+\frac{\left(k_{1}^{p}+k_{2}^{p}\right) n T^{p(\alpha-1)+1}}{p(p(\alpha-1)+1)} .
$$

Setting

$$
v(t)=\widetilde{k}+\sum_{i=1}^{n} \frac{1}{q} \int_{0}^{t}\left(\gamma_{i}(s) g(s) u(s)\right)^{q} d s+\sum_{i=1}^{n} \frac{1}{q} \int_{0}^{t}\left(\lambda_{i}(s) f_{i}(s)\right)^{q}\left(\psi_{i}(u(s))\right)^{q} d s,
$$

we see that

$$
v^{\prime}(t)=\sum_{i=1}^{n} \frac{1}{q}\left(\gamma_{i}(t) g(t) u(t)\right)^{q}+\sum_{i=1}^{n} \frac{1}{q}\left(\lambda_{i}(t) f_{i}(t)\right)^{q}\left(\psi_{i}(u(t))\right)^{q}, \text { and } v(0)=\widetilde{k} .
$$

Since the $\psi_{i}$ are nondecreasing and $u(t) \leq v(t)$,

$$
v^{\prime}(t) \leq \sum_{i=1}^{n} \frac{1}{q}\left(\gamma_{i}(t) g(t) v(t)\right)^{q}+\sum_{i=1}^{n} \frac{1}{q}\left(\lambda_{i}(t) f_{i}(t)\right)^{q}\left(\psi_{i}(v(t))\right)^{q},
$$

so

$$
v^{\prime}(t) \leq m(t)\left(v^{q}(t)+\widetilde{\psi}(v(t))\right),
$$

where

$$
m(t)=\max \left(\sum_{i=1}^{n} \frac{1}{q}\left(\gamma_{i}(t) g(t)\right)^{q}, \sum_{i=1}^{n} \frac{1}{q}\left(\lambda_{i}(t) f_{i}(t)\right)^{q}\right) \text { and } \widetilde{\psi}(v)=\max \left\{\psi_{i}^{q}(v): i=1,2, \ldots, n\right\}
$$


Therefore,

$$
\frac{v^{\prime}(t)}{v^{q}(t)+\widetilde{\psi}(v(t))} \leq m(t), \quad t \in[0, T]
$$

Integrating the above inequality from 0 to $t$, we obtain

$$
\int_{0}^{t} \frac{v^{\prime}(s)}{v^{q}(s)+\widetilde{\psi}(v(s))} d s \leq \int_{0}^{t} m(s) d s,
$$

or

$$
\int_{v(0)}^{v(t)} \frac{d x}{x^{q}+\widetilde{\psi}(x)} \leq \int_{0}^{t} m(s) d s
$$

Now

$$
\int_{v(0)}^{v(t)} \frac{d x}{x^{q}+\widetilde{\psi}(x)}=\int_{k_{0}}^{v(t)} \frac{d x}{x^{q}+\widetilde{\psi}(x)}-\int_{k_{0}}^{\widetilde{k}} \frac{d x}{x^{q}+\widetilde{\psi}(x)}=\Psi(v(t))-\Psi(\widetilde{k})
$$

since $v(0)=\widetilde{k}$. Thus, it follows that

$$
\Psi(v(t)) \leq \Psi(\widetilde{k})+\int_{0}^{t} m(s) d s,
$$

and hence

$$
v(t) \leq \Psi^{-1}\left(\Psi(\widetilde{k})+\int_{0}^{t} m(s) d s\right) .
$$

Since $u(t) \leq v(t)$, we obtain

$$
u(t) \leq \Psi^{-1}\left(\Psi(\widetilde{k})+\int_{0}^{t} m(s) d s\right)
$$

which proves the theorem.

In the particular case where $g(t) \equiv 0$, we have the following corollary to Theorem 1 .

Corollary 1. Let $k_{0}, k_{1}>0, q>\frac{1}{\alpha}$, and the functions $f_{i}, \lambda_{i}, \psi_{i}$, and $u$ be as in Theorem 1 . If

$$
u(t) \leq k_{0}+\sum_{i=1}^{n} k_{1} \int_{0}^{t}(t-s)^{\alpha-1} f_{i}(s) \lambda_{i}(s) \psi_{i}(u(s)) d s,
$$

then

$$
u(t) \leq \Psi^{-1}\left(\Psi(\bar{k})+\sum_{i=1}^{n} \frac{1}{q} \int_{0}^{t}\left(f_{i}(s) \lambda_{i}(s)\right)^{q} d s\right)
$$

where

$$
\Psi(z)=\int_{k_{0}}^{z} \frac{d y}{\widetilde{\psi}(y)}, \widetilde{\psi}(y)=\max \left\{\psi_{i}^{q}(y): i=1,2, \ldots, n\right\}, \bar{k}=k_{0}+\frac{n k_{1}^{p} T^{p(\alpha-1)+1}}{p(p(\alpha-1)+1)},
$$

$\Psi^{-1}$ is the inverse function of $\Psi$, and for every $t \in[0, T]$,

$$
\Psi(\bar{k})+\sum_{i=1}^{n} \frac{1}{q} \int_{0}^{t}\left(f_{i}(s) \lambda_{i}(s)\right)^{q} d s \in \operatorname{Dom}\left(\Psi^{-1}\right) .
$$

Proof. Proceeding exactly as in the proof of Theorem 1, we again arrive at (3). Define the function $\widetilde{\psi}$ by

$$
\widetilde{\psi}(x)=\max \left\{\psi_{i}^{q}(x): i=1,2 \ldots, n\right\},
$$


where $\frac{1}{p}+\frac{1}{q}=1$. Then,

$$
v^{\prime}(t) \leq \sum_{i=1}^{n} \frac{1}{q}\left(f_{i}(t) \lambda_{i}(t)\right)^{q} d s \widetilde{\psi}(v(t))
$$

which implies that

$$
\frac{v^{\prime}(t)}{\widetilde{\psi}(v(t))} \leq \sum_{i=1}^{n} \frac{1}{q}\left(f_{i}(t) \lambda_{i}(t)\right)^{q} d s .
$$

Integrating the above inequality from 0 to $t$ gives

$$
\int_{v(0)}^{v(t)} \frac{d y}{\widetilde{\psi}(y)} \leq \sum_{i=1}^{n} \frac{1}{q} \int_{0}^{t}\left(f_{i}(s) \lambda_{i}(s)\right)^{q} d s .
$$

Therefore,

$$
v(t) \leq \Psi^{-1}\left(\Psi(\bar{k})+\sum_{i=1}^{n} \frac{1}{q} \int_{0}^{t}\left(f_{i}(s) \lambda_{i}(s)\right)^{q} d s\right) .
$$

In view of the fact that $u(t) \leq v(t)$, this proves the corollary.

If $\alpha=1$ and $f_{i}(t) \equiv 1$ for $i=1,2, \ldots, n$ in Corollary 1 , we have the following version of the result of Agarwal et al. ([9], Theorem 2.1).

Corollary 2. Let $k_{0}, k_{1}>0$, and the functions $\lambda_{i}, \psi_{i}$, and $u$ be as in Theorem 1. If

$$
u(t) \leq k_{0}+\sum_{i=1}^{n} k_{1} \int_{0}^{t} \lambda_{i}(s) \psi_{i}(u(s)) d s
$$

then

$$
u(t) \leq \Psi_{n}^{-1}\left(\Psi_{n}\left(k_{n-1}\right)+\int_{0}^{t} \lambda_{n}(s) d s\right),
$$

where

$$
\Psi_{i}(z)=\int_{z_{i}}^{z} \frac{d y}{\psi_{i}(y)}, z>z_{i}>0, i=1,2, \ldots, n,
$$

the constants $k_{i}$ are given by

$$
k_{i}=\Psi_{i}^{-1}\left[\Psi_{i}\left(k_{i-1}\right)+\left\|\lambda_{i}\right\|_{T_{1}}\right],
$$

and $T_{1} \in I$ is the largest number such that

$$
\left\|\lambda_{i}\right\|_{T_{1}}:=\int_{0}^{T_{1}} \lambda_{i}(s) d s \leq \int_{k_{i-1}}^{\infty} \frac{d y}{\psi_{i}(y)} d s .
$$

For $n=1, g \equiv 0$, and $f_{1} \equiv 1 \equiv \lambda_{1}$ in Theorem 1 , we obtain the following fractional Bihari type inequality on bounded intervals.

Corollary 3. Let $k_{0}, k_{1}>0, q>\frac{1}{\alpha}$, and the functions $\psi$ and $u$ be as in Theorem 1 such that

$$
u(t) \leq k_{0}+k_{1} \int_{0}^{t}(t-s)^{\alpha-1} \psi(u(s)) d s .
$$

Then, for $t \in[0, T]$, we have

$$
u(t) \leq \Phi^{-1}\left(\Phi(\bar{k})+\frac{t}{q}\right)
$$


where

$$
\frac{1}{p}+\frac{1}{q}=1, \quad \Phi(z)=\int_{k_{0}}^{z} \frac{d z}{(\psi(z))^{q}}, \quad \bar{k}=k_{0}+\frac{k_{1}^{p} T^{p(\alpha-1)+1}}{p(p(\alpha-1)+1)},
$$

and

$$
\Phi(\bar{k})+\frac{t}{q} \in \operatorname{Dom}\left(\Phi^{-1}\right) .
$$

If $n=1$ and $\lambda_{1}(t) \equiv 1$ in Corollary 1 , then by using Hölder's inequality, we have the following result.

Corollary 4. Let $k_{0}, k_{1}>0$, the functions $f, \psi$, and $u$ be as in Theorem $1, p\left(1-\frac{1}{q}\right)=1$, and $q>\frac{1}{\alpha}$. If

$$
u(t) \leq k_{0}+k_{1} \int_{0}^{t}(t-s)^{\alpha-1} f(s) \psi(u(s)) d s,
$$

then

$$
u(t) \leq\left[\Psi^{-1}\left(\frac{2^{q} k_{1}^{q} T^{q(p(\alpha-1)+1)}}{(p(\alpha-1)+1)^{q}} \int_{0}^{t} f^{q}(s) d s\right)\right]^{\frac{1}{q}}, t \in[0, T],
$$

where

$$
\Psi(z)=\int_{2^{q} k^{q}}^{z} \frac{d x}{(\psi(\sqrt[q]{x}))^{q}}, \quad z \geq 2^{q} k^{q} .
$$

Next, for convenience, we introduce a class of functions to be used to obtain new fractional Bihari type inequalities.

Definition 1. A function $\psi:[0, \infty) \rightarrow[0, \infty)$ is said to belong to the class $H$ if: $\left(H_{1}\right) \psi$ is positive, continuous, and nondecreasing for $z>0$.

$\left(H_{2}\right)$ There exists a continuous function $\phi:[0, \infty) \rightarrow[0, \infty)$ such that

$$
\psi(\gamma z) \leq \phi(\gamma) \psi(z), \quad \text { for every } z \geq 0 \text { and } \gamma>0 .
$$

For additional details on this class of functions, see [26].

Theorem 2. Let $0<\alpha<1, q\rangle \frac{1}{\alpha}$, and assume that for $i=1,2, \ldots, n$, the functions $f, \lambda_{i}, \gamma_{i}, h$, and $g$ are non-negative and locally integrable, $f$ and $g$ are increasing, and $(f g) / h$ is decreasing on $I=[0, T]$. Additionally, let $\psi_{i} \in H, i=1,2, \ldots, n$, with corresponding multiplier functions $\phi_{i}$. If $u(t)$ is a non-negative continuous function on I satisfying

$$
u(t) \leq h(t)+g(t) \sum_{i=1}^{n} \int_{0}^{t}(t-s)^{\alpha-1} \gamma_{i}(s) u(s) d s+f(t) \sum_{i=1}^{n} \int_{0}^{t}(t-s)^{\alpha-1} \lambda_{i}(s) \psi_{i}(u(s)) d s,(6)
$$

then

$$
u(t) \leq h(t) \Psi^{-1}\left(\Psi(\widetilde{k})+\int_{0}^{t} m(s) d s\right)
$$

where

$$
\begin{gathered}
m(t)=\max \left(\sum_{i=1}^{n} \frac{1}{q}\left(\gamma_{i}(t) g(t)\right)^{q}, \sum_{i=1}^{n} \frac{1}{q}\left(\frac{f(t) \lambda_{i}(t) \phi_{i}(h(t))}{h(t)}\right)^{q}\right), \widetilde{k}=1+\frac{2 n T^{p(\alpha-1)+1}}{p(p(\alpha-1)+1)} \\
\Psi(z)=\int_{1}^{z} \frac{d y}{y^{q}+\widetilde{\psi}(y)} \text {, and } \widetilde{\psi}(y)=\max \left\{\psi_{i}^{q}(y), i=1, \ldots, n\right\}
\end{gathered}
$$


Proof. From (6), we have

$$
\frac{u(t)}{f(t) g(t)} \leq \frac{h(t)}{g(t) f(t)}+\sum_{i=1}^{n} \int_{0}^{t}(t-s)^{\alpha-1} \frac{\gamma_{i}(s)}{f(s)} u(s) d s+\sum_{i=1}^{n} \int_{0}^{t}(t-s)^{\alpha-1} \frac{\lambda_{i}(s)}{g(s)} \psi_{i}(u(s)) d s,
$$

which implies that

$$
\begin{aligned}
\frac{u(t)}{h(t)} \leq & 1+\sum_{i=1}^{n} \int_{0}^{t}(t-s)^{\alpha-1} \frac{g(s) \gamma_{i}(s)}{h(s)} u(s) d s+\sum_{i=1}^{n} \int_{0}^{t}(t-s)^{\alpha-1} \frac{f(s) \lambda_{i}(s)}{h(s)} \psi_{i}(u(s)) d s \\
\leq & 1+\sum_{i=1}^{n} \int_{0}^{t}(t-s)^{\alpha-1} g(s) \gamma_{i}(s) \frac{u(s)}{h(s)} d s \\
& +\sum_{i=1}^{n} \int_{0}^{t}(t-s)^{\alpha-1} \frac{f(s) \lambda_{i}(s) \phi_{i}(h(s))}{h(s)} \psi_{i}\left(\frac{u(s)}{h(s)}\right) d s .
\end{aligned}
$$

With $z(t)=\frac{u(t)}{h(t)}$, we see that this last inequality is equivalent to

$z(t) \leq 1+\sum_{i=1}^{n} \int_{0}^{t}(t-s)^{\alpha-1} g(s) \gamma_{i}(s) z(s) d s+\sum_{i=1}^{n} \int_{0}^{t}(t-s)^{\alpha-1} \frac{f(s) \lambda_{i}(s) \phi_{i}(h(s))}{h(s)} \psi_{i}(z(s)) d s$.

If we replace $f_{i}$ by $\frac{f(s) \phi_{i}(h(s))}{h(s)}$ in Theorem 1 , we obtain

$$
z(t) \leq \Psi^{-1}\left(\Psi(\widetilde{k})+\int_{0}^{t} m(s) d s\right) .
$$

Therefore,

$$
u(t) \leq h(t) \Psi^{-1}\left(\Psi(\widetilde{k})+\int_{0}^{t} m(s) d s\right)
$$

which completes the proof.

For $n=1, g \equiv 0$, and $f(t) \equiv k$, we next have a another fractional Bihari type inequality on a bounded interval.

Corollary 5. Let $q>\frac{1}{\alpha}, u, \lambda:[0, \infty) \rightarrow[0, \infty)$ be continuous functions, $\psi \in H$ have corresponding multiplier function $\phi$ on $[0, \infty), h(t)>0$ be a nondecreasing continuous function on $[0, \infty)$, and $k>0$ be a constant. If

$$
u(t) \leq h(t)+k \int_{0}^{t}(t-s)^{\alpha-1} \lambda(s) \psi(u(s)) d s, t \in[0, T],
$$

then

$$
u(t) \leq h(t) \Psi^{-1}\left(\Psi(\bar{k})+\frac{1}{q} \int_{0}^{t}\left(\frac{\phi(h(s)) \lambda(s)}{h(s)}\right)^{q} d s\right), \quad t \in[0, T]
$$

where

$$
\Psi(z)=\int_{1}^{z} \frac{d u}{(\psi(u))^{q}} d u, \quad \bar{k}=1+\frac{k^{p} T^{p(\alpha-1)+1}}{p(p(\alpha-1)+1)^{\prime}}
$$

and

$$
\Psi(\bar{k})+\frac{1}{q} \int_{0}^{t}\left(\frac{\phi(h(s)) f(s)}{h(s)}\right)^{q} d s \in \operatorname{Dom}\left(\Psi^{-1}\right), \quad t \in[0, T] .
$$


Proof. From (7) and the fact that $h$ is nondecreasing,

$$
\begin{aligned}
\frac{u(t)}{h(t)} & \leq 1+k \int_{0}^{t}(t-s)^{\alpha-1} \frac{\lambda(s)}{h(s)} \psi\left(\frac{h(s) u(s)}{h(s)}\right) d s \\
& \leq 1+k \int_{0}^{t}(t-s)^{\alpha-1} \frac{\phi(h(s)) \lambda(s)}{h(s)} \psi\left(\frac{u(s)}{h(s)}\right) d s .
\end{aligned}
$$

Applying Young's inequality gives

$$
\begin{aligned}
\frac{u(t)}{h(t)} & \leq 1+\frac{k^{p}}{p} \int_{0}^{t}(t-s)^{p(\alpha-1)} d s+\frac{1}{q} \int_{0}^{t}\left(\frac{\phi(h(s)) \lambda(s)}{h(s)}\right)^{q}\left(\psi\left(\frac{u(s)}{h(s)}\right)\right)^{q} d s \\
& \leq 1+\frac{k^{p} T^{p(\alpha-1)+1}}{p(p(\alpha-1)+1)}+\frac{1}{q} \int_{0}^{t}\left(\frac{\phi(h(s)) \lambda(s)}{h(s)}\right)^{q}\left(\psi\left(\frac{u(s)}{h(s)}\right)\right)^{q} d s
\end{aligned}
$$

where $\frac{1}{p}+\frac{1}{q}=1$. Define

$$
v(t)=1+\frac{k^{p} T^{p(\alpha-1)+1}}{p(p(\alpha-1)+1)}+\frac{1}{q} \int_{0}^{t}\left(\frac{\phi(h(s)) \lambda(s)}{h(s)}\right)^{q}\left(\psi\left(\frac{u(s)}{h(s)}\right)\right)^{q} d s .
$$

It follows that

$$
v^{\prime}(t)=\frac{1}{q}\left(\frac{\phi(h(t)) \lambda(t)}{h(t)}\right)^{q}\left(\psi\left(\frac{u(t)}{h(t)}\right)\right)^{q}, \quad v(0)=\bar{k} .
$$

Since $\psi$ is nondecreasing,

$$
v^{\prime}(t) \leq \frac{1}{q}\left(\frac{\phi(h(t)) \lambda(t)}{h(t)}\right)^{q}(\psi(v(t)))^{q} .
$$

An integration from 0 to $t$ yields

$$
\int_{\bar{k}}^{v(t)} \frac{d z}{(\psi(z))^{q}} \leq \frac{1}{q} \int_{0}^{t}\left(\frac{\phi(h(s)) \lambda(s)}{h(s)}\right)^{q} d s .
$$

Therefore,

$$
v(t) \leq \Psi^{-1}\left(\Psi(\bar{k})+\frac{1}{q} \int_{0}^{t}\left(\frac{\phi(h(s)) \lambda(s)}{h(s)}\right)^{q} d s\right), \quad t \in[0, T],
$$

and so

$$
u(t) \leq h(t) \Psi^{-1}\left(\Psi(\bar{k})+\frac{1}{q} \int_{0}^{t}\left(\frac{\phi(h(s)) \lambda(s)}{h(s)}\right)^{q} d s\right), \quad t \in[0, T],
$$

which completes the proof.

As another result in this same spirit, we have the following theorem.

Theorem 3. Let $0<\alpha<1, q>\frac{1}{\alpha}$, and assume that $u, \lambda_{i}$, and $h$ are non-negative functions, $i=1,2, \ldots, n$, that are locally integrable on $I=[0, T]$, and let $\psi_{i} \in H$ with corresponding multiplier functions $\phi_{i}$. In addition, assume that the function $h$ is nondecreasing and satisfies

$(\widetilde{H})$ for each $t \in I$ there exists a continuous function $\chi$ such that $h(z(t)) \leq \frac{\chi(t)}{z(t)}$.

If

$$
u(t) \leq h(u(t))+\sum_{i=1}^{n} \int_{0}^{t}(t-s)^{\alpha-1} \lambda_{i}(s) \frac{u(s)}{\phi_{i}(h(u(s)))} \psi_{i}(u(s)) d s,
$$


then

$$
u(t) \leq\left[\chi(t) \Psi^{-1}\left(\Psi(\widetilde{k})+\int_{0}^{t} m(s) d s\right)\right]^{\frac{1}{2}},
$$

where

$$
\begin{gathered}
m(t)=\max \left(\frac{n}{p q}, \sum_{i=1}^{n} \frac{1}{q^{2}} \lambda_{i}^{q^{2}}(t)\right), \widetilde{k}=1+\frac{n T^{p(\alpha-1)+1}}{p(p(\alpha-1)+1)} \\
\Psi(z)=\int_{1}^{z} \frac{d y}{y^{p q}+\widetilde{\psi}(y)} \text {, and } \widetilde{\psi}(y)=\max \left\{\psi_{i}^{q^{2}}(y): i=1,2, \ldots, n\right\} .
\end{gathered}
$$

Proof. From (8) and the facts that $\Psi$ satisfies $\left(\mathrm{H}_{2}\right)$ and $h$ is increasing, we obtain

$$
\frac{u(t)}{h(u(t))} \leq 1+\sum_{i=1}^{n} \int_{0}^{t}(t-s)^{\alpha-1} \lambda_{i}(s) \frac{u(s)}{h(u(s))} \psi_{i}\left(\frac{u(s)}{h(u(s))}\right) d s .
$$

With $z(t)=\frac{u(t)}{h(u(t))}$, this becomes

$$
z(t) \leq 1+\sum_{i=1}^{n} \int_{0}^{t}(t-s)^{\alpha-1} \lambda_{i}(s) z(s) \psi_{i}(z(s)) d s .
$$

By Young's inequality,

$$
\begin{aligned}
z(t) & \leq 1+\sum_{i=1}^{n}\left(\frac{1}{p} \int_{0}^{t}(t-s)^{p(\alpha-1)}+\frac{1}{q} \int_{0}^{t} \lambda_{i}^{q}(s) z^{q}(s) \psi_{i}^{q}(z(s))\right) d s \\
& \leq 1+\frac{n T^{p(\alpha-1)+1}}{p(p(\alpha-1)+1)}+\sum_{i=1}^{n} \frac{1}{q}\left(\frac{1}{p} \int_{0}^{t} z(s)^{p q} d s+\frac{1}{q} \int_{0}^{t} \lambda_{i}^{q^{2}}(s) \psi_{i}^{q^{2}}(z(s))\right) d s \\
& \leq \widetilde{k}+\sum_{i=1}^{n} \frac{1}{p q} \int_{0}^{t} z^{p q}(s) d s+\sum_{i=1}^{n} \frac{1}{q^{2}} \int_{0}^{t} \lambda_{i}^{q^{2}}(s) \psi_{i}^{q^{2}}(z(s)) d s,
\end{aligned}
$$

where $\widetilde{k}=1+\frac{n T^{p(\alpha-1)+1}}{p(p(\alpha-1)+1)}$.

Letting

$$
v(t)=\widetilde{k}+\sum_{i=1}^{n} \frac{1}{p q} \int_{0}^{t} z^{p q}(s) d s+\sum_{i=1}^{n} \frac{1}{q^{2}} \int_{0}^{t} \lambda_{i}^{q^{2}}(s) \psi_{i}^{q^{2}}(z(s)) d s,
$$

we define the functions $m$ and $\widetilde{\psi}$ by

$$
m(t)=\max \left(\frac{n}{p q}, \sum_{i=1}^{n} \frac{1}{q^{2}} \lambda_{i}^{q^{2}}(t)\right) \text { and } \widetilde{\psi}(t)=\max \left\{\psi_{i}^{q^{2}}(t): i=1, \ldots, n\right\} .
$$

Since the $\psi_{i}$ are nondecreasing,

$$
v^{\prime}(t)=\frac{n}{p q} z^{p q}(t)+\sum_{i=1}^{n} \frac{1}{q^{2}} \lambda_{i}^{q^{2}}(t) \psi_{i}^{q^{2}}(z(t)) \leq m(t)\left(v^{p q}(t)+\widetilde{\psi}(v(t))\right) .
$$

Hence,

$$
\frac{v^{\prime}(t)}{v^{p q}(t)+\widetilde{\psi}(v(t))} \leq m(t)
$$

and integrating from 0 to $t$, we obtain

$$
\int_{v(0)}^{v(t)} \frac{d x}{x^{p q}+\widetilde{\psi}(x)} \leq \int_{0}^{t} m(s) d s .
$$


With $v(0)=\widetilde{k}$, we have

$$
\Psi(v(t)) \leq \Psi(\widetilde{k})+\int_{0}^{t} m(s) d s,
$$

which implies that

$$
v(t) \leq \Psi^{-1}\left(\Psi(\widetilde{k})+\int_{0}^{t} m(s) d s\right) .
$$

Since $\frac{u(t)}{h(u(t))}=z(t) \leq v(t)$ and $(\widetilde{H})$ holds, we finally obtain

$$
u(t) \leq h(u(t)) \Psi^{-1}\left(\Psi(\widetilde{k})+\int_{0}^{t} m(s) d s\right) \leq \frac{\chi(t)}{u(t)} \Psi^{-1}\left(\Psi(\widetilde{k})+\int_{0}^{t} m(s) d s\right) .
$$

Thus,

$$
u^{2}(t) \leq \chi(t) \Psi^{-1}\left(\Psi(\widetilde{k})+\int_{0}^{t} m(s) d s\right)
$$

and so

$$
u(t) \leq\left[\chi(t) \Psi^{-1}\left(\Psi(\widetilde{k})+\int_{0}^{t} m(s) d s\right)\right]^{\frac{1}{2}} .
$$

This completes the proof of the theorem.

\section{Multivalued Analysis}

In order to apply the inequalities obtained above to fractional differential inclusions, we need to recall some basic notions from multivalued analysis (see, for example, [27,28])

For any set $X$, we employ the following notation.

$$
\begin{aligned}
& \mathcal{P}(X)=\{Y \subset X: Y \neq \varnothing\} ; \\
& \mathcal{P}_{c l}(X)=\{Y \in \mathcal{P}(X): Y \text { closed }\} \\
& \mathcal{P}_{b}(X)=\{Y \in \mathcal{P}(X): Y \text { bounded }\} ; \\
& \mathcal{P}_{c v}(X)=\{Y \in \mathcal{P}(X): Y \text { convex }\} ; \\
& \mathcal{P}_{c p}(X)=\{Y \in \mathcal{P}(X): Y \text { compact }\} ; \\
& \mathcal{P}_{w c p}(X)=\{Y \in \mathcal{P}(X): Y \text { weakly compact }\} .
\end{aligned}
$$

A multi-valued map $G: X \longrightarrow \mathcal{P}(X)$ has convex (closed) values if $G(x)$ is convex (closed) for all $x \in X$. We say that $G$ is bounded on bounded sets if $G(B)$ is bounded in $X$ for each bounded set $B \subset X$, i.e., $\sup _{x \in B}\{\sup \{|y|: y \in G(x)\}\}<\infty$.

Definition 2. ([28], Definition 2.30) A multifunction $F: X \rightarrow \mathcal{P}(Y)$ is said to be upper semicontinuous (weakly upper semi-continuous) at the point $x_{0} \in X$ if for every open $W \subseteq Y(W$ weakly open) such that $F\left(x_{0}\right) \subset W$, there exists a neighborhood $V\left(x_{0}\right)$ (weakly open) of $x_{0}$ such that $F\left(V\left(x_{0}\right)\right) \subset W$. A multifunction is called upper semi-continuous (u.s.c.) on $X$ if it is u.s.c. at $x$ for each $x \in X$.

Definition 3. ([28], Definition 2.30) A multifunction $F: X \rightarrow \mathcal{P}(Y)$ is lower-continuous at the point $x_{0} \in X$ if for every open $W \subseteq Y$ such that $F\left(x_{0}\right) \cap W \neq \varnothing$, there exists a neighborhood $V\left(x_{0}\right)$ of $x_{0}$ such that $F(x) \cap W \neq \varnothing$ for all $x \in V\left(x_{0}\right)$. A multifunction is lower semi-continuous (l.s.c.) provided that is lower semi-continuous at every point $x \in X$.

Definition 4. ([27], Definition 2.1.1) A mapping $G: X \rightarrow Y$ is closed if the graph $\mathcal{G} r(G)$ is a closed subset of $X \times Y$, i.e., for sequences $\left(x_{n}\right)_{n \in \mathbb{N}} \subset X$ and $\left(y_{n}\right)_{n \in \mathbb{N}} \subset Y$, if $x_{n} \rightarrow x_{*}$ and $y_{n} \rightarrow y_{*}$ as $n \rightarrow \infty$ with $y_{n} \in G\left(x_{n}\right)$, then $y_{*} \in G\left(x_{*}\right)$. 
A mapping $G$ is said to be completely continuous if it is u.s.c. and for every bounded set $A \subseteq X, G(A)$ is relatively compact, i.e., there exists a relatively compact set $K \subset X$ such that $G(A)=\bigcup\{G(x): x \in A\} \subset K$. Additionally, $G$ is compact if $G(X)$ is relatively compact and it is called locally compact if for each $x \in X$, there exists $U \in \mathcal{V}(x)$ such that $G(U)$ is relatively compact.

Definition 5. ([27], Definition 2.6.2) Let $X$ be a metric space and $E$ be a Banach space. A multivalued map $F: X \rightarrow \mathcal{P}(E)$ is upper hemicontinuous if for each $x^{*} \in E^{*}$, the function $\sigma\left(x^{*}, F(\cdot)\right): X \rightarrow \mathbb{R} \cup\{+\infty\}$ given by

$$
\sigma\left(x^{*}, F(x)\right)=\sup _{y \in F(x)}\left\langle x^{*}, y\right\rangle
$$

is upper semicontinuous.

Next, we recall the following results.

Lemma 1. ([29], Proposition 1.2) If $G: X \rightarrow \mathcal{P}_{c l}(Y)$ is u.s.c., then $\mathcal{G} r(G)$ is a closed subset of $X \times Y$. Conversely, if $G$ is locally compact, has nonempty compact values, and a closed graph, then it is u.s.c.

Lemma 2. ([30]) If $F: X \rightarrow \mathcal{P}_{w c p, c v}(E)$ is upper hemicontinuous, then it is weakly u.s.c.

Lemma 3. ([30]) If $F: X \rightarrow \mathcal{P}_{w c p, c v}(E)$ is weakly u.s.c. if and only if for sequences $\left(x_{n}\right)_{n \in \mathbb{N}} \subset X$ and $\left(y_{n}\right)_{n \in \mathbb{N}} \subset E$, if $x_{n} \rightarrow x_{*}$, as $n \rightarrow \infty$ with $y_{n} \in F\left(x_{n}\right)$, then there is a subsequence $y_{n_{k}} \rightarrow y_{*} \in F\left(x_{*}\right)$.

Theorem 4. ([31]) Let $X$ be a reflexive Banach space and $F: X \rightarrow \mathcal{P}_{c l, c v}(X)$ be a upperhemicontinuous multivalued map. Let $J$ be a finite interval of $\mathbb{R}$ and the sequences $\left(x_{n}: J \rightarrow X\right)_{n \in \mathbb{N}}$ and $\left(y_{n}: J \rightarrow X\right)_{n \in \mathbb{N}}$ satisfy the following conditions:

1) $\left(x_{n}\right)_{n \in \mathbb{N}}$ converges to a function $x: J \rightarrow X$;

2) $\left(y_{n}\right)_{n \in \mathbb{N}}$ converges weakly to $y \in L^{p}(J, E), 1 \leq p<\infty$;

3) $y_{n}(t) \in \overline{c o} B\left(F\left(B\left(x_{n}(t), \epsilon_{n}\right), \epsilon_{n}\right)\right)$ for a.e. all $t \in J$, where $\epsilon_{n} \rightarrow 0$ as $n \rightarrow \infty$.

Then $y(t) \in F(x(t))$ for a.e. $t \in J$.

Definition 6. ([28], Definition 9.8) A space $A$ is closed acyclic if:

(a) $H_{0}(A)=\mathbb{Q}$;

(b) $H_{n}(A)=0$ for every $n>0$, where $H_{*}=\left\{H_{n}\right\}_{n \geq 0}$ is the Čech-homology functor with compact carriers and coefficients in the field of rationals $\mathbb{Q}$.

That is, a space $A$ is acyclic if the map $j:\{p\} \rightarrow X$, with $j(p)=x_{0} \in A$, induces an isomorphism $j_{*}: H_{*}(\{p\}) \rightarrow H_{*}(A)$.

Remark 1. If $R_{\delta}-$ is a compact connected space that is acyclic with respect to the Čech-homology functor, then it has the same homology as a one-point space.

Definition 7. ([28], Definition 9.9) A u.s.c. map $F: X \rightarrow \mathcal{P}(Y)$ is called acyclic if for each $x \in X, F(x)$ is compact acyclic.

Definition 8. ([28], Definition 9.15) Let $X$ be a metric space and E be a Banach space. A map $N: X \rightarrow E$ is proper if it is continuous and for every compact $K \subset E$ the set $N^{-1}(K)$ is compact.

Definition 9. ([28], Definition 2.29) A map L $: X \rightarrow Y$ is said to be a Vietoris map if the following conditions are satisfied:

(i) $L: X \rightarrow Y$ is proper; 
(ii) L is surjective;

(iii) the set $L^{-1}(y)$ is acyclic for every $y \in Y$.

It is clear that any multivalued operator $F: X \rightarrow \mathcal{P}(Y)$ admits the standard factorization through the graph $\mathcal{G} r(F)$, i.e., there exists a diagram

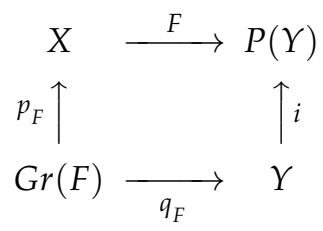

where $p_{F}: \mathcal{G} r(F) \rightarrow X$ and $q_{F}: \mathcal{G} r(F) \rightarrow Y$ are the projections such that

$$
F(x)=q_{F}\left(p_{F}^{-1}(x)\right) \text { for all } x \in X .
$$

This suggests the following definition and properties.

Definition 10. ([32], Definition 40.1) A multivalued operator $F: X \rightarrow \mathcal{P}(Y)$ is called admissible if there exists a metric space $\widetilde{X}$ and two continuous maps $\widetilde{p}: \widetilde{X} \rightarrow X$ and $\widetilde{q}: \widetilde{X} \rightarrow Y$ such that

(i) $\tilde{p}$ is a Vietoris map, and

(ii) $F(x)=\widetilde{q}\left(\widetilde{p}^{-1}(x)\right)$ for any $x \in X$.

Proposition 1. ([32]) If $F: X \rightarrow \mathcal{P}(Y)$ and $G: Y \rightarrow \mathcal{P}(Z)$ are two admissible maps, then the composition $G \circ F$ is admissible.

Given a Banach space $(X,\|\cdot\|)$, for a multivalued map $F: J \times X \rightarrow \mathcal{P}(X)$, set

$$
\|F(t, x)\|_{\mathcal{P}}:=\sup \{\|v\|: v \in F(t, x)\} .
$$

A multivalued map $G: J \longrightarrow \mathcal{P}_{c p}(X)$ is said to be measurable if for each $x \in \mathbb{R}$ the function $Y: J \longrightarrow \mathbb{R}$ defined by

$$
Y(t)=d(x, G(t))=\inf \{\|x-z\|: z \in G(t)\}
$$

is measurable.

A multifunction $F:[a, b] \rightarrow \mathcal{P}_{c p}(X)$ is strongly measurable if there exists a sequence $\left\{F_{n}: n \in \mathbb{N}\right\}$ of step multifunctions such that

$$
H_{d}\left(F_{n}(t), F(t)\right) \rightarrow 0 \text { as } n \rightarrow \infty \text { for } \mu \text { a.e } t \in[a, b],
$$

where $\mu$ denotes a Lebesgue measure on $[a, b]$ and $H_{d}$ is the Hausdorff metric on $\mathcal{P}_{c p}(X)$.

In what follows, $L^{p}([a, b], X)$ denotes the Banach space of functions $y: J \longrightarrow X$, that are Bochner integrable with norm

$$
\|y\|_{p}=\left(\int_{a}^{b}\|y(t)\|^{p} d t\right)^{\frac{1}{p}}, \quad p \in[1, \infty) .
$$

For each $y \in C(J, X)$, the set

$$
S_{F, y}=\left\{f \in L^{1}(J, X): f(t) \in F(t, y(t)) \text { for a.e. } t \in[0, b]\right\}
$$

is known as the set of selection functions for $F$.

Lemma 4. ([32], Theorem 19.7) Let X be a separable metric space and $G$ be a multivalued map with nonempty closed values. Then $G$ has a measurable selection. 
Definition 11. ([28], Definition 2.80) A multivalued map $F$ is a Carathéodory function if

(a) the function $t \mapsto F(t, x)$ is strong measurable for each $x \in X$;

(b) for a.e. $t \in J$, the map $x \mapsto F(t, x)$ is upper semi-continuous.

Furthermore, $F$ is $L^{1}$-Carathéodory if it is locally integrably bounded, i.e., for each positive $r$, there exists $h_{r} \in L^{1}\left(J, \mathbb{R}^{+}\right)$such that

$$
\|F(t, x)\|_{\mathcal{P}} \leq h_{r}(t), \text { for a.e. } t \in J \text { and all }\|x\| \leq r .
$$

Measures of Noncompactness (MNC)

For more details on measure of noncompactness than given below, we refer the reader to [33-35] and the references therein.

Definition 12. ([28], Definition 2.91) Let E be a Banach space and $(\mathcal{A}, \geq)$ be a partially ordered set. A map $\beta: \mathcal{P}(X) \rightarrow \mathcal{A}$ is called a measure of noncompactness on $X(M N C)$, if

$$
\beta(\overline{c o} \Omega)=\beta(\Omega)
$$

for every $\Omega \in \mathcal{P}(X)$.

Notice that if $D$ is dense in $\Omega$, then $\overline{c o} \Omega=\overline{c o} D$ and hence

$$
\beta(\Omega)=\beta(D) .
$$

Definition 13. ([28], Definition 2.92) A measure of noncompactness $\beta$ is:

(a) Monotone if $\Omega_{0}, \Omega_{1} \in \mathcal{P}(X)$ with $\Omega_{0} \subset \Omega_{1}$, implies $\beta\left(\Omega_{0}\right) \leq \beta\left(\Omega_{1}\right)$.

(b) Nonsingular if $\beta(\{a\} \cup \Omega)=\beta(\Omega)$ for every $a \in X, \Omega \in \mathcal{P}(X)$.

(c) Invariant with respect to the union with compact sets if $\beta(K \cup \Omega)=\beta(\Omega)$ for every relatively compact set $K \subset X$, and $\Omega \in \mathcal{P}(X)$.

(d) Real if $\mathcal{A}=\overline{\mathbb{R}}_{+}=[0, \infty]$ and $\beta(\Omega)<\infty$ for every bounded $\Omega$.

(e) Semi-additive if $\beta\left(\Omega_{0} \cup \Omega_{1}\right)=\max \left(\beta\left(\Omega_{0}\right), \beta\left(\Omega_{1}\right)\right)$ for every $\Omega_{0}, \Omega_{1} \in \mathcal{P}(X)$.

(f) Regular if the condition $\beta(\Omega)=0$ is equivalent to the relative compactness of $\Omega$.

As an example of a $M N C$, consider the Hausdorff $M N C$

$$
\chi(\Omega)=\inf \{\varepsilon>0: \Omega \text { has a finite } \varepsilon-n e t\} .
$$

Recall that a bounded set $A \subset X$ has a finite $\varepsilon$-net if there exits a finite subset $S \subset X$ such that $A \subset S+\varepsilon \bar{B}$ where $\bar{B}$ is a closed ball in $X$. Other examples are given by the following measures of noncompactness defined on the space of continuous functions $C([0, b], X)$ with values in a Banach space $X$ :

(i) the modulus of fiber noncompactness

$$
\varphi(\Omega)=\sup _{t \in[0, b]} \chi_{X}(\Omega(t)),
$$

where $\chi_{X}$ is the Hausdorff $M N C$ in $X$ and $\Omega(t)=\{y(t): y \in \Omega\}$.

(ii) the modulus of equicontinuity

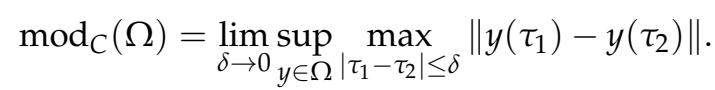

It should be mentioned that these $M N C$ satisfy all above-mentioned properties except regularity. 
Definition 14. ([28]) Let $\mathcal{M}$ be a closed subset of a Banach space $X$ and $\beta: \mathcal{P}(X) \rightarrow(\mathcal{A}, \geq)$ be a $M N C$ on $E$. A multivalued map $\mathcal{F}: \mathcal{M} \rightarrow \mathcal{P}_{c p}(X)$ is said to be $\beta$-condensing if for every $\Omega \subset \mathcal{M}$, the relation

$$
\beta(\Omega) \leq \beta(\mathcal{F}(\Omega))
$$

implies the relative compactness of $\Omega$.

Some important results on fixed point theory with $M N C$ s are recalled next (see, for example, [34] for proofs and additional details). The first one is a compactness criterion.

Lemma 5. ([34], Theorem 5.1.1) Let $N: L^{1}([a, b], X) \rightarrow C([a, b], X)$ be an operator satisfying the following conditions:

$\left(\mathcal{S}_{1}\right) N$ is $\xi$-Lipschitz: there exists $\xi>0$ such that for every $f, g \in L^{1}([a, b], X)$,

$$
\|N f(t)-N g(t)\| \leq \xi \int_{a}^{b}\|f(s)-g(s)\| d s, \text { for all } t \in[a, b] .
$$

$\left(\mathcal{S}_{2}\right) \mathrm{N}$ is weakly-strongly sequentially continuous on compact subsets: for any compact $K \subset X$ and any sequence $\left\{f_{n}\right\}_{n=1}^{\infty} \subset L^{1}([a, b], X)$ such that $\left\{f_{n}(t)\right\}_{n=1}^{\infty} \subset K$ for a.e. $t \in[a, b]$, the weak convergence of $f_{n} \rightarrow f_{0}$ implies the strong convergence of $N\left(f_{n}\right) \rightarrow N\left(f_{0}\right)$ as $n \rightarrow+\infty$.

Then for every semi-compact sequence $\left\{f_{n}\right\}_{n=1}^{\infty} \subset L^{1}([0, b], X)$, the image sequence $N\left(\left\{f_{n}\right\}_{n=1}^{\infty}\right)$ is relatively compact in $C([a, b], X)$.

Lemma 6. ([34], Theorem 5.2.2]) Let an operator $N: L^{1}([a, b], X) \rightarrow C([a, b], X)$ satisfy conditions $\left(\mathcal{S}_{1}\right)-\left(\mathcal{S}_{2}\right)$ together with

$\left(\mathcal{S}_{3}\right)$ There exits $\eta \in L^{1}([a, b])$ such that for every integrably bounded sequence $\left\{f_{n}\right\}_{n=1}^{\infty}$, we have

$$
\chi\left(\left\{f_{n}(t)\right\}_{n=1}^{\infty}\right) \leq \eta(t), \text { for a.e. } t \in[a, b],
$$

where $\chi$ is the Hausdorff MNC.

Then

$$
\chi\left(\left\{N\left(f_{n}\right)(t)\right\}_{n=1}^{\infty}\right) \leq 2 \xi \int_{a}^{b} \eta(s) d s, \text { for all } t \in[a, b],
$$

where $\xi$ is the constant in $\left(\mathcal{S}_{1}\right)$.

\section{Fixed Point Theory}

We begin with some basics of fixed point theory.

Theorem 5. ([32]) Let $D \subset X$ be a nonempty closed convex and bounded subset of $X$ and $N: D \rightarrow \mathcal{P}(D)$ an $\beta$-condensing admissible multivalued operator, where $\gamma$ is a nonsingular measure of noncompactness defined on subsets of $X$. Then FixN is nonempty and compact.

The next result is concerned with the nonlinear alternative for $\beta$-condensing $u$.s.c. multi-valued maps.

Lemma 7. Let $V \subset X$ be a bounded open neighborhood of zero and $N: \bar{V} \rightarrow \mathcal{P}(X)$ be a $\beta$ admissible multi-valued map, where $\beta$ is a nonsingular measure of noncompactness defined on subsets of $X$, that satisfies the Leray-Schauder boundary condition

$$
x \notin \lambda N(x)
$$

for all $x \in \partial V$ and $0<\lambda<1$. Then Fix $N$ is nonempty and compact.

Proof. Let $C$ be the set defined by

$$
C=\{x \in \bar{V}: x \in \lambda N(x) \text { for some } \lambda \in[0,1]\} .
$$


It is clear that $C$ is nonempty set since $0 \in C$. To show that $C$ is closed, let $\left(x_{n}\right)_{n \in \mathbb{N}} \subset C$ be a sequence converging to $x$; then there exists $\left(\lambda_{n}\right)_{n \in \mathbb{N}} \subset[0,1]$ such that

$$
x_{n} \in \lambda_{n} N\left(x_{n}\right) \text { for } n \in \mathbb{N} .
$$

Since $[0,1]$ is compact, there exists a subsequence of $\left(\lambda_{n}\right)_{n \in \mathbb{N}}$ that converges to $\lambda \in$ $[0,1]$.

If $\lambda \in(0,1]$, then using the fact that $N$ is admissible, we see that $N$ has a closed graph, so $x \in \lambda N(x)$. that

If $\lambda=0$, it is clear that $N\left(\overline{\left\{x_{n}: n \in \mathbb{N}\right\}}\right)$ is a compact set, so there exists $M>0$ such

$$
\|y\| \leq M \text { for any } y \in N\left(\overline{\left\{x_{n}: n \in \mathbb{N}\right\}}\right) \text {. }
$$

This implies

$$
\left\|x_{n}\right\| \leq \lambda_{n} M, \quad n \in \mathbb{N},
$$

that is, $x=0 \in 0 N(0)$, and we conclude that $C$ is closed set in $X$.

Since $C \cap X \backslash V=\varnothing$, Urysohn's lemma guarantees the existence of a continuous function $\mu: X \rightarrow[0,1]$ such that $\mu(x)=1$ for $x \in C$ and $\mu(x)=0$ for $x \in X \backslash V$. Let $r: X \rightarrow \bar{V}$ be a retraction of the space $X$ onto $\bar{V}$.

We introduce the multivalued operator $\widetilde{N}: X \rightarrow \mathcal{P}(X)$ defined by

$$
\widetilde{N}(x)= \begin{cases}\mu(x) N(x), & x \in \bar{V}, \\ \{0\}, & x \in X \backslash V .\end{cases}
$$

Observe that

$$
\widetilde{N}(x)=f \circ(\mu(x) \times N(r(x))), \quad x \in X,
$$

where $f:[0,1] \times X \rightarrow X$ is defined by

$$
f(\lambda, x)=\lambda x, \quad x \in X .
$$

Let $C_{*}=\overline{c o}(N(\bar{V}) \cup\{0\})$; it is easy to show that

$$
\widetilde{N}\left(C_{*}\right) \subset C_{*} .
$$

It follows from the definition of $\widetilde{N}$ that it is an admissible multivalued map.

Next, we show that $\widetilde{N}$ is $\beta$-condensing. Let $D \in \mathcal{P}_{b}\left(C_{*}\right)$ be such that

$$
\beta(D) \leq \widetilde{N}(D) .
$$

If $D \cap \bar{V}=\varnothing$, then from the definition of $\widetilde{N}$, we have

$$
\beta(D) \leq \beta(\widetilde{N}(D)) \leq \beta(\{0\})=0,
$$

so $D$ is relatively compact. On the other hand, if $D \cap \bar{V} \neq \varnothing$, then

$$
\beta(D \cap \bar{V}) \leq \beta(D) \leq \beta(\widetilde{N}(D)) \leq \beta(\widetilde{N}(D \cap \bar{V})) .
$$

From the definition of $\widetilde{N}$,

$$
\beta(\widetilde{N}(D \cap \bar{V}))=\beta(\mu(D \cap \bar{V}) N(D \cap \bar{V}))=\beta\left(\bigcup_{x \in D \cap \bar{V}} \mu(x) N(D \cap \bar{V})\right) .
$$


From Definition 13, for every $x \in X$, we have

$$
\beta(\mu(x) N(D \cap \bar{V}))=|\mu(x)| \beta(N(D \cap \bar{V})) \leq \beta(N(D \cap \bar{V})) .
$$

Hence,

$$
\beta(D \cap \bar{V}) \leq \beta(D) \leq \beta(\widetilde{N}(D)) \leq \beta(N(D \cap \bar{V})) .
$$

Since $N$ is $\beta$-condensing, we obtain that $D \cap \bar{V}$ is relatively compact. Moreover, since $N$ is u.s.c., $\beta(D)=0$, so $D$ is relatively compact. Therefore, $\widetilde{N}$ is $\beta$-admissible since it is admissible and $\beta$-condensing.

We now use Theorem 5 to show that $\widetilde{N}$ has at least one fixed point $x \in C_{*}$ such that $x \in \widetilde{N}(x)$. Since $0 \in V, x \in \bar{V}$ and $x \in \mu(x) N(x)$. Hence, $x \in C$. This implies that $x \in N(x)$ so $\operatorname{Fix}(N) \neq \varnothing$.

To see that $\operatorname{Fix}(N)$ is compact, first note that

$$
\operatorname{Fix}(N) \subset N(\operatorname{Fix}(N)) \text {. }
$$

Therefore,

$$
\beta(\operatorname{Fix}(N)) \leq \beta(N(\operatorname{Fix}(N))) .
$$

Since $N$ is $\beta$-admissible, we conclude that $\operatorname{Fix}(N)$ is compact. This completes the proof of the lemma.

An additional result on the set of fixed points of $F$ is contained in the following proposition.

Proposition 2. Let $X$ be a Banach space and $N: X \rightarrow \mathcal{P}(X)$ be a $\beta$-admissible multivalued map, where $\beta$ is a nonsingular measure of noncompactness defined on subsets of $X$. If the set

$$
\mathcal{M}=\{x \in X: x \in \lambda N(x), \text { for some } \lambda \in(0,1)\}
$$

is bounded, then FixN $\neq \varnothing$ and is compact.

Proof. Since $\mathcal{M}$ is a bounded set, there exists $M>0$ such that

$$
\|x\|<M \text { for each } x \in \mathcal{M} \text {. }
$$

We can easily prove that $N: \overline{B(0, M)} \rightarrow \mathcal{P}(X)$ is a $\beta$-admissible operator and

$$
x \notin \lambda N(x) \text { for all } x \in \partial \overline{B(0, M)} .
$$

Consequently, from Lemma 7, the set $\operatorname{Fix}(N) \neq \varnothing$ and is compact.

Similarly, we have the following result.

Lemma 8. ([34]) Let $W$ be a closed subset of a Banach space $E$ and $N: W \rightarrow \mathcal{P}_{c p}(X)$ be a closed $\beta$-condensing multivalued map, where $\beta$ is a nonsingular measure of noncompactness defined on subsets of $X$. If the fixed point set Fix $(N)$ is bounded, then it is compact.

In what follows, we wish to replace the Leray-Schauder boundary condition with the following "star-shaped" condition.

Definition 15. ([27], Definition 4.2.7) Let X be a Banach space. An open bounded neighborhood of the origin, $V$, is strictly star-shaped with respect to the origin if for any $x \in \partial V$, we have

$$
\{\lambda x: \lambda>0\} \cap \partial V=\{x\} .
$$


Remark 2. If $V$ is a bounded open neighborhood of the origin, the following strict inclusions hold (see ([36], Proposition 1)):

$$
\text { Convex } \subset \text { Strictly Star-shaped } \subset \text { Star-shaped. }
$$

For any strictly star-shaped open and bounded neighborhood $V$ of the origin, we can define the Minkowski function $\mu_{V}: X \rightarrow[0, \infty)$ by

$$
\mu_{V}(x)=\inf \{\lambda>0: x \in \lambda V\} \text {. }
$$

This function satisfies the properties:

- $\quad \mu_{v}(t x)=t \mu(x), \quad$ for all $x \in X$ and $t \geq 0$.

- $\mu_{V}(x)>0$ for any $x \in X$.

- $V=\left\{x: \mu_{V}(x)<1\right\}, \bar{V}=\left\{x: \mu_{V}(x) \leq 1\right\}$, and $\partial V=\left\{x: \mu_{V}(x)=1\right\}$.

Remark 3. For a strictly star set, the Minkowski function can equivalently be defined as: $\mu(0)=0$ and, for $x \neq 0, \mu_{V}(x)$ is the unique positive number such that $\frac{x}{\mu_{V}(x)} \in V$.

Proposition 3. ([37]) Let X be a Banach space and $V$ be an open bounded neighborhood of zero. If $V$ is strictly star-shaped, then the Minkowski function $\mu_{V}$ is continuous and the mapping $R_{V}: X \rightarrow \bar{V}$ given by

$$
R_{V}(x)= \begin{cases}\frac{x}{\mu_{V}(x)}, & x \in X \backslash \bar{V}, \\ x, & x \in \bar{V},\end{cases}
$$

is a continuous retract of $X$ into closure of $V$.

In 2005, Jiménez-Melado and Morales [36] introduced the so-called interior condition for single valued maps. In the following definition, we introduce a multivalued version of this condition.

Definition 16. ([36], Page 501) Let $X$ be a real Banach space and $V$ be open subset of $X$ with $0 \in V$. We say that the multivalued map $N: \bar{V} \rightarrow \mathcal{P}(X)$ satisfies the interior condition if there exists $\delta>0$ such that

$$
\lambda x \notin N(x) \text { for } x \in V_{\delta}, \lambda>1 \text {, and } N(x) \cap X \backslash \bar{V} \neq \varnothing \text {, }
$$

where

$$
V_{\delta}=\{x \in V: d(x, \partial V)<\delta\}
$$

The following result is taken from González, Jiménez-Melado, and Llorens-Fuster ([37], Proposition 2).

Proposition 4. Let $X$ be a Banach space and $V$ be a strictly star-shaped open bounded neighborhood of the origin. Let $0 \leq k \leq \widetilde{K}$, where $k=d(0, \partial V)$ and $\widetilde{K}=\sup \{\|x\|: x \in \partial V\}$, and choose $\delta \in(0, k]$. Define $L_{V}^{\delta}: X \rightarrow X$ by

$$
L_{V}^{\delta}(x)= \begin{cases}\frac{r+(1-r) \mu_{V}(x)}{\mu_{V}^{2}(x)} x, & x \in X \backslash \bar{V}, \\ x, & x \in \bar{V} .\end{cases}
$$

Then $L_{V}^{\delta}$ is continuous, $L_{V}^{\delta}(X \backslash \bar{V}) \subset V_{\delta}$, and $L_{V}^{\delta}(x) \in \overline{c o}(\{0\} \cup\{x\})$ for all $x \in X$.

The following fixed point theorem is for multivalued maps satisfying the interior condition. 
Theorem 6. Let $X$ be a real Banach space and let $V$ be a bounded open and strictly star-shaped subset of $X$ with $0 \in V$. If the multivalued map $N: \bar{V} \rightarrow \mathcal{P}(X)$ is $\beta$-condensing, admissible, and satisfies the interior condition, then $N$ has at least one fixed point.

Proof. Let $R_{V}: X \rightarrow \bar{V}$ be a retraction of $X$ into $\bar{V}$. Define the multivalued operator $N_{*}: \bar{V} \rightarrow \mathcal{P}(X)$ by

$$
N_{*}(x)= \begin{cases}\frac{r+(1-r) \mu_{V}(x)}{\mu_{V}^{2}(x)} N\left(R_{V}(x)\right), & x \in X \backslash \bar{V}, \\ N(x), & x \in \bar{V} .\end{cases}
$$

We can write $N_{*}$ as

$$
\widetilde{N}_{*}(x)=g \circ\left(\widetilde{\mu}(x) \times N\left(R_{V}(x)\right)\right), \quad x \in X,
$$

where $g:[0,1] \times X \rightarrow X$ is defined by

$$
g(\lambda, x)=\lambda x, \quad x \in X,
$$

and

$$
\widetilde{\mu}(x)= \begin{cases}\frac{r+(1-r) \mu_{V}(x)}{\mu_{V}^{2}(x)}, & x \in X \backslash \bar{V}, \\ 1, & x \in \bar{V},\end{cases}
$$

are continuous functions. Then, by Proposition $1, N_{*}$ is an admissible operator, and we see that $\overline{c o}(N(\bar{V}) \cup\{0\})$ is invariant under $N_{*}$. Let $D \in \mathcal{P}_{b}(\bar{V})$; then from the definition of $N_{*}$,

$$
\beta\left(N_{*}(D)\right)=\beta(N(D)) .
$$

Therefore,

$$
\beta\left(N_{*}(D)\right) \leq \beta(N(D)) \text { for all } D \in \mathcal{P}_{b}(\bar{V}) .
$$

From this it follows that $N_{*}$ is a $\beta$-condensing admissible operator. We wish to show that the conditions of Mönch's theorem [38] hold. Let $D \subset \bar{V}$ with $D \subset \overline{c o}\left(\{0\} \cup N_{*}(D)\right)$; then

$$
\beta(D) \leq \beta\left(\overline{c o}\left(\{0\} \cup N_{*}(D)\right)\right)=\beta\left(\overline{c o}\left(N_{*}(D)\right)\right) .
$$

Hence,

$$
\beta(D) \leq \beta\left(N_{*}(D)\right) .
$$

Using the fact that $N_{*}$ is $\beta$-condensing, we see that $D$ is relatively compact.

Next, we show that $N_{*}$ satisfies the Leray-Schauder condition. Since $N$ satisfies the interior condition, there exists $\delta \in I:=(0, \widetilde{K})$ such that

$$
\lambda x \notin N(x) \quad \text { for } x \in V_{\delta}, \lambda>1 \text {, and } N(x) \cap X \backslash \bar{V} \neq \varnothing \text {, }
$$

where

$$
\widetilde{K}=\sup \{\|x\|: x \in \partial V\} .
$$

As in the proof of ([36], Theorem 1), for any $t \in\left(1-\frac{\delta}{\widetilde{K}^{K}}, 1\right)$, the set $V_{t}=\{t x: x \in V\}$ is an open subset of $V, \overline{t V}=t \bar{V}$, and $t \bar{V} \subset V$.

Additionally, we can easily show that

$$
\bigcup_{t \in I} \bar{V}_{t}=V
$$

Thus,

$$
\partial V \subset \bigcup_{t \in I} \partial \bar{V}_{t} \subset \bigcup_{t \in I} \partial V_{t}
$$


Now suppose there exist $\lambda_{t}>1$ and $x_{t} \in \partial V_{t}$ such that $\lambda_{t} x_{t} \in N_{*}\left(x_{t}\right)$. Then by the definition of $N_{*}$,

$$
\lambda_{t} x_{t} \in N\left(x_{t}\right), \quad \lambda_{t}>1, \text { and } x_{t} \in \partial V_{t} .
$$

Since $V$ is strictly star-shaped and $x_{t} \in \partial V_{t} \subset V$, there exists a unique $z_{t} \in \partial V$ such that $x_{t}=t z_{t}$ (see Remark 3). Hence, we obtain

$$
d\left(x_{t}, \partial V\right) \leq\left\|x_{t}-z_{t}\right\| \leq(1-t) \widetilde{K}<\delta,
$$

and consequently, by the interior condition, we have $\lambda_{t} x_{t} \in \bar{V}$. This implies that $\lambda_{t} x_{t} \in$ $\left[x_{t}, z_{t}\right]$ since that is a strictly star-shaped set. Thus, we conclude that

$$
\left\|x_{t}\right\| \leq\left\|\lambda_{t} x_{t}\right\| \leq\left\|z_{t}\right\| \leq \widetilde{K}
$$

Therefore, the set

$$
D_{*}=\left\{x_{t}: x_{t} \text { satisfies the relation (10), } t \in I\right\}
$$

is bounded and $D_{*} \subset \overline{c o}\left\{N\left(D_{*}\right) \cup\{0\}\right\}$. Since $N$ is $\beta$-condensing, $D_{*}$ is relatively compact, and hence there exists a sequence $\left(x_{n}\right)_{n \in \mathbb{N}}$ converging to $x$ in $\bar{V}$ and a sequence $\left(\lambda_{n}\right)_{n \in \mathbb{N}}$ in $\mathbb{R}$ such that

$$
\lambda_{n} x_{n} \in N\left(x_{n}\right), \lambda_{n} \rightarrow 1, t_{n} \rightarrow 1 \text {, and } x_{n} \rightarrow x \text { as } n \rightarrow \infty .
$$

It is easy to see that (11), implies $\lim _{n \rightarrow \infty} x_{n}=\lim _{n \rightarrow \infty} z_{n}=x$ and there exists $n \in \mathbb{N}$ such that $x_{n} \in V$. We then have that

$$
d\left(x_{n}, \partial V\right)<\delta \text { implies } x_{n} \in V_{\delta} .
$$

The interior condition of $N$ implies that

$$
\lambda_{n} x_{n} \notin N\left(x_{n}\right) \text { and } N\left(x_{n}\right) \cap X \backslash \bar{V}=\varnothing,
$$

which contradicts (10).

Therefore, $N_{*}$ satisfies the Leray-Schauder condition. Since $\bar{V}$ is a retract of $X, N_{*}$ is an admissible map and satisfies Mönch's theorem. Then, by ([39], Theorem 5), there exists $x \in \bar{V}$ such that $x \in N_{*}(x)$. From the definition of $N_{*}$, it follows that $x \in N(x)$, and this completes the proof of the theorem.

\section{Fractional Differential Inclusions with Delay}

In this section we wish to apply some of the inequalities obtained in Section 2 and the new Leray-Schauder type fixed point theorem obtained in Section 4 to proving the existence of solutions to the Cauchy problem for the fractional delay differential inclusion

$$
\begin{cases}{ }^{c} D^{\alpha} x(t) \in F\left(t, x_{t}\right), & t \in J, \\ x(t)=\varphi(t), & t \in J_{0},\end{cases}
$$

where $0<\alpha \leq 1, J:=[0, T], J_{0}:=[-r, 0]$, and $F: J \times C\left(J_{0}, E\right) \rightarrow \mathcal{P}_{c p, c v}(E)$ is a multivalued function. For any function $x$ defined on $[-r, T]$ and any $t \in J$, we denote by $x_{t}$ the element of $C(I, E)$ defined by

$$
x_{t}(\theta)=x(t+\theta), \theta \in I .
$$

Here $x_{t}(\cdot)$ represents the history of the state from time $t-r$ up to the present time $t$. The study of differential inclusions has emerged as an important area of research due to their applicability to problems in optimal control theory and other areas; see, for example, the monographs $[40,41]$ and the references contained therein. Additionally, differential inclusions can incorporate differential equations with discontinuities in the right hand 
side (or even for the case where the right hand side is inaccurately known) [34,42-44]. Additional background on differential inclusions and multivalued analysis can be found in [27-29,32,45-48]. For recent results on the existence of solutions to fractional differential equations and inclusions with various types of delays, we refer the reader to [49-55].

We begin by recalling the definitions of a fractional integral and the Caputo fractional derivative. Here, $\Gamma$ denotes the well-known gamma function.

Definition 17. ([49], Definition 1.4) The fractional integral of order $\beta>0$ with lower limit 0 for a function $f$ is defined as

$$
I^{\beta} f(t)=\frac{1}{\Gamma(\beta)} \int_{0}^{t}(t-s)^{\beta-1} f(s) d s, t>0,
$$

provided the right-hand side is pointwise defined on $[0, \infty)$.

Definition 18. ([49], Definition 1.6) The Caputo fractional derivative of order $\beta$ with $0 \leq$ $n-1<\beta<n$ and lower limit 0 for a function $f$ is given by

$$
{ }^{c} D^{\beta} f(t)=\frac{1}{\Gamma(n-\beta)} \int_{0}^{t}(t-s)^{n-\beta-1} f(s) d s=I^{n-\beta} f(t), t>0,0 \leq n-1<\beta<n .
$$

We will need to make use of the following assumptions in our results in this section.

$\left(\mathcal{H}_{1}\right)$ The mulivalued map $F(t, \cdot)$ has a strong measurable selection for $u \in C\left(J_{0}, E\right)$.

$\left(\mathcal{H}_{2}\right)$ The map $F(t, \cdot)$ is upper hemicontinuous for almost all $t \in J$.

$\left(\mathcal{H}_{3}\right)$ There exist functions $\psi \in H$ and $f \in L^{q}\left(J, \mathbb{R}^{+}\right)$with $q>\frac{1}{\alpha}$ such that

$$
\|F(t, u)\| \leq f(t) \psi(\|u\|)
$$

for every $t \in J$ and $u \in C\left(J_{0}, E\right)$.

$\left(\mathcal{H}_{4}\right)$ There exists $g \in L^{q}\left(J, \mathbb{R}^{+}\right)$such that for all bounded $D \in C\left(J_{0}, E\right)$, we have

$$
\alpha(F(t, D)) \leq g(t) \sup _{\theta \in J_{0}} \alpha(D(\theta)) \text {, for a.e. } t \in J_{0}
$$

where

$$
D(\theta)=\{\phi(\theta): \phi \in D\} .
$$

In the following proposition we establish some properties of the selection function operator.

Proposition 5. If $E$ is a reflexive space and the multifunction $F$ satisfies conditions $\left(\mathcal{H}_{1}\right)-\left(\mathcal{H}_{3}\right)$ for $1<q<\infty$, then the selection function operator $S_{F}^{q}: C([-r, T], E) \rightarrow L^{q}([-r, T], E)$ given by

$$
S_{F}^{q}(x)=\left\{v \in L^{q}([0, T], E): v(t) \in F\left(t, x_{t}\right) \text { a.e. } t \in J\right\}
$$

has nonempty convex weakly compact values and is a weakly upper semicontinuous multivalued operator.

Proof. The convexity of $S_{F}^{q}$ follows immediately from the convexity of $F$. Let $x \in C([-r, T]$, $E)$; there exists a step sequence $\left(x_{n}\right)$ converging uniformly to $x$ in $[-r, T]$. By conditions $\left(\mathcal{H}_{1}\right)$ and $\left(\mathcal{H}_{3}\right)$, there is a sequence $\left(v_{n}\right)$ of strong measurable selections of $F$ such that

$$
v_{n}(t) \in F\left(t,\left(x_{n}\right)_{t}\right) \quad \text { a.e. } t \in J
$$

and

$$
\left\|v_{n}(t)\right\| \leq f(t) \psi(\rho) \text { a.e. } t \in J
$$

where

$$
\left\|x_{n}\right\|_{\infty} \leq \rho \quad \text { for all } n \in \mathbb{N} \text {. }
$$


Hence,

$$
v_{n} \in S_{F}^{q}\left(x_{n}\right) \text { for each } n \in \mathbb{N},
$$

and $\left(v_{n}\right)$ is bounded sequence in $L^{q}(J, E)$. Since $E$ is a reflexive space, by the duality theorem ([56], Theorem V.1.1), the space $L^{q}(J, E)$ is also reflexive. By the Eberlein-S̆mulian Theorem (see Brezis [57]), there exists a subsequence, still denoted by $\left(v_{n}\right)$, that converges weakly to $v \in L^{q}(J, E)$. Additionally, we have

$$
v(t) \in \bigcap_{n \geq 1} \overline{\left\{v_{k}(t): k \geq n\right\}} \subset \bigcap_{n \geq 1} \overline{c o}\left\{v_{k}(t): k \geq n\right\}, \text { a.e. } t \in J .
$$

Since $F(t, \cdot)$ is upper hemicontinuous,

$$
\limsup _{n \rightarrow \infty} \sigma\left(x^{*}, F\left(t,\left(x_{n}\right)_{t}\right)\right) \leq \sigma\left(x^{*}, F\left(t, x_{t}\right)\right), \text { for all } x^{*} \in E^{*} .
$$

From (13) it follows that

$$
v_{n}(t) \in \overline{c o} B\left(F\left(t,\left(x_{n}\right) t\right), \frac{1}{n}\right) \text { a.e. } t \in J .
$$

By (14)-(16), and applying the Lebesgue dominated convergence theorem, we obtain

$$
v(t) \in F\left(t, x_{t}\right), \quad \text { a.e. } t \in J .
$$

Therefore, $S_{F}^{q}(x) \neq \varnothing$ and $S_{F}^{q}(x) \in \mathcal{P}_{w c p, c v}(C([-r, T], E))$.

Let $\left\{\left(x_{n}, v_{n}\right)\right\}_{n \in \mathbb{N}} \in \operatorname{Gr}\left(S_{F}^{q}\right)$ such that $\left(x_{n}\right)$ converges to $x \in C([-r, T], E)$. Then, for each $n \in \mathbb{N}$, we have

$$
v_{n}(t) \in F\left(t,\left(x_{n}\right)_{t}\right) \text {, a.e. } t \in J .
$$

Applying a similar argument, we can prove that the Nemytskii operator is weakly upper semicontinuous.

Now consider the operator $N: C([-r, T], E) \rightarrow \mathcal{P}(C([-r, T], E))$ defined by

$$
N(x)(t)=\left\{h \in C([-r, T], E): h(t)=\left\{\begin{array}{ll}
\varphi(t), & \mathbf{t} \in J_{0}, \\
\varphi(0)+\frac{1}{\Gamma(\alpha)} \int_{0}^{t}(t-s)^{\alpha-1} v(s) d s, & \mathbf{t} \in J,
\end{array}\right\},\right.
$$

where $v \in S_{F}^{q}(x)$. Notice that $N$ can be written in the form

$$
N(x)=L(x)+\left(K \circ S_{F}^{q}\right)(x), \quad x \in C([-r, T], E),
$$

where $L: C(\mid-r, T], E) \rightarrow C([-r, T], E)$ is defined by

$$
L(x(t))= \begin{cases}\varphi(t), & \mathrm{t} \in J_{0}, \\ \varphi(0), & \mathrm{t} \in J,\end{cases}
$$

and $K: L^{q}([0, T], E) \rightarrow C([-r, T], E)$ by

$$
K(v(t))= \begin{cases}0, & \mathrm{t} \in J_{0}, \\ \frac{1}{\Gamma(\alpha)} \int_{0}^{t}(t-s)^{\alpha-1} v(s) d s, & \mathrm{t} \in J .\end{cases}
$$

Next, we establish an important property of the operator $N(x)$ defined in (17).

Proposition 6. If conditions $\left(\mathcal{H}_{1}\right)-\left(\mathcal{H}_{4}\right)$ hold, then the operator $N$ is u.s.c. and $\beta$-condensing.

Proof. We will divide the proof into several steps. We begin by showing that $N$ is $u$.s.c. and has nonempty convex values. 
Step 1: $N(\cdot) \in \mathcal{P}_{c v}(E)$. Since $L$ is single valued, $K$ is a bounded linear operator, and $S_{F}^{q}$ has nonempty convex values, for each $x \in C([-r, T], E)$ we have $N(x) \in \mathcal{P}_{c v}(E)$.

Step 2: $N$ is u.s.c. We first show that $N$ maps bounded sets into bounded subsets of $C([-r, T], E)$. Let $B_{\rho}:=\left\{x \in C([-r, T], E):\|x\|_{\infty} \leq \rho\right\}$. For $x \in B_{\rho}$ and $h \in N(x)$, there exists $v \in S_{F, x}$ such that

$$
h(t)= \begin{cases}\varphi(t), & \mathrm{t} \in J_{0}, \\ \varphi(0)+\frac{1}{\Gamma(\alpha)} \int_{0}^{t}(t-s)^{\alpha-1} v(s) d s, & \mathbf{t} \in J .\end{cases}
$$

Then, by $\left(\mathcal{H}_{3}\right)$ and Hölder's inequality

$$
\begin{aligned}
\|h(t)\| & \leq\|\varphi(0)\|+\left\|\frac{1}{\Gamma(\alpha)} \int_{0}^{t}(t-s)^{\alpha-1} v(s) d s\right\| \\
& \leq\|\varphi\|_{\infty}+\frac{1}{\Gamma(\alpha)} \int_{0}^{t}(t-s)^{\alpha-1} f(s) \psi\left(\left\|x_{s}\right\|\right) d s \\
& \leq\|\varphi\|_{\infty}+\frac{1}{\Gamma(\alpha)} \int_{0}^{t}(t-s)^{\alpha-1} f(s) \psi\left(\|x\|_{\infty}\right) d s \\
& \leq\|\varphi\|_{\infty}+\frac{\psi(\rho)}{\Gamma(\alpha)}\left(\int_{0}^{t}(t-s)^{p \alpha-p} d s\right)^{\frac{1}{p}}\|f\|_{L^{q}} .
\end{aligned}
$$

Hence,

$$
\|h\|_{\infty} \leq\|\varphi\|_{\infty}+\frac{T^{\alpha-1+\frac{1}{p}} \psi(\rho)}{\Gamma(\alpha)}\|f\|_{L^{q}} .
$$

Next, we wish to show that $N$ maps bounded sets into equicontinuous subsets of $C([-r, T], E)$. Let $t_{1}, t_{2} \in J$ with $t_{1}<t_{2}$ and let $B_{\rho}$ be a bounded subset of $C([-r, T], E)$. If $x \in B_{\rho}$, then for each $h \in N(x)$, we have

$$
\left\|h\left(t_{2}\right)-h\left(t_{1}\right)\right\| \leq \frac{\psi(\rho)}{\Gamma(\alpha)} \int_{t_{1}}^{t_{2}}\left(t_{2}-s\right)^{\alpha-1} f(s) d s+\frac{\psi(\rho)}{\Gamma(\alpha)} \int_{0}^{t_{1}}\left|\left(t_{2}-s\right)^{\alpha-1}-\left(t_{1}-s\right)^{\alpha-1}\right| f(s) d s .
$$

From the Hölder and Biernacki inequalities, it follows that

$$
\begin{aligned}
\| h\left(t_{2}\right) & -h\left(t_{1}\right) \| \\
& \leq \frac{p \psi(\rho)\left(t_{2}-t_{1}\right)^{\alpha-1+\frac{1}{p}}}{(p \alpha-p+1) \Gamma(\alpha)}\|f\|_{L^{q}}+\frac{\psi(\rho)}{\Gamma(\alpha)}\left(\int_{0}^{t_{1}}\left(\left(t_{1}-s\right)^{\alpha-1}-\left(t_{2}-s\right)^{\alpha-1}\right)^{p} d s\right)^{\frac{1}{p}}\|f\|_{L^{q}} \\
& \leq \frac{p \psi(\rho)\left(t_{2}-t_{1}\right)^{\alpha-1+\frac{1}{p}}}{(p \alpha-p+1) \Gamma(\alpha)}\|f\|_{L^{q}}+\frac{\psi(\rho)}{\Gamma(\alpha)}\left(\int_{0}^{t_{1}}\left(\left(t_{1}-s\right)^{p \alpha-p}-\left(t_{2}-s\right)^{p \alpha-p}\right) d s\right)^{\frac{1}{p}}\|f\|_{L^{q}} \\
& \leq \frac{p \psi(\rho)\left(t_{2}-t_{1}\right)^{\alpha-1+\frac{1}{p}}}{(p \alpha-p+1) \Gamma(\alpha)}\|f\|_{L^{q}}+\frac{\psi(\rho)\left(t_{1}^{p \alpha-p+1}+\left(t_{2}-t_{1}\right)^{p \alpha-p+1}-t_{2}^{p \alpha-p+1}\right)^{\frac{1}{p}}}{(p \alpha-p+1) \Gamma(\alpha)}\|f\|_{L^{q}} .
\end{aligned}
$$

The left-hand side tends to zero as $t_{2}-t_{1} \rightarrow 0$, so $N\left(B_{\rho}\right)$ is equicontinuous in $C([-r, T], E)$.

Step 3: $N$ is a condensing operator for a suitable MNC $\gamma$. Given a bounded subset $D \in \mathcal{P}_{b}([-r, T], E)$, let $\bmod _{C}(D)$ be the modulus of equicontinuity of the set of functions $D$, i.e.,

$$
\bmod _{C}(D)=\lim _{\delta \rightarrow 0} \sup _{x \in D} \max _{\left|\tau_{2}-\tau_{1}\right| \leq \delta}\left|x\left(\tau_{1}\right)-x\left(\tau_{2}\right)\right| .
$$

It is well known (see, for example, ([34], Example 2.1.2)) that $\bmod _{C}(D)$ defines an $M N C$ in $C([-r, T], E)$ that satisfies all of the properties in Definition 13 except regularity. 
Given the Hausdorff MNC $\chi$, let $\bar{\gamma}$ be the real MNC defined on bounded subsets of $C([-r, T], X)$ by

$$
\bar{\gamma}(D)=\sup _{t \in[0, T]} e^{-\tau \int_{0}^{t} f^{q}(s) d s} \chi(D(t))
$$

where $\tau>0$ chosen such that

$$
q_{*}=\frac{2 T^{\alpha-1+\frac{1}{p}}}{\sqrt[p]{q \tau(p \alpha-p+1)} \Gamma(\alpha)}<1 .
$$

Then we define the $M N C$ on bounded subsets of $C([-r, T], E)$ by

$$
\gamma(D)=\max _{D \in \Delta(C([-r, T], E))}\left(\bar{\gamma}(D), \bmod _{C}(D)\right),
$$

where $\Delta(C([-r, T], E))$ is the collection of all countable subsets of $B$. The $M N C \gamma$ is monotone, regular, and nonsingular (see ([34], Example 2.1.4)). To show that $N$ is $\gamma$ condensing, let $B \subset C([-r, T], E)$ be a bounded set in $C([-r, T], E)$ such that

$$
\gamma(B) \leq \gamma(N(B)) \text {. }
$$

To show that $B$ is relatively compact, let $\left\{x_{n}: n \in \mathbb{N}\right\} \subset B$. From (18), each $h_{n}$ in $N\left(x_{n}\right)$ can be represented as

$$
h_{n}=L\left(x_{n}\right)+K\left(v_{n}\right), \text { with } v_{n} \in S_{F}\left(x_{n}\right) .
$$

Moreover, (20) yields

$$
\gamma\left(\left\{h_{n}: n \in \mathbb{N}\right\}\right) \geq \gamma\left(\left\{x_{n}: n \in \mathbb{N}\right\}\right) .
$$

From condition $\left(\mathcal{H}_{4}\right)$, for a.e. $t \in[0, T]$,

$$
\begin{aligned}
\chi\left(\left\{v_{n}(t): n \in \mathbb{N}\right\}\right) \leq \chi( & F\left(t,\left\{x_{n}(t)\right\}_{n=1}^{\infty}\right) \leq g(t) \chi\left(\left\{\left(x_{t}\right)_{n}\right\}_{n=1}^{\infty}\right) \\
& \leq g(t) \sup _{0 \leq s \leq t} \chi\left(\left\{\left(x_{s}\right)_{n}\right\}_{n=1}^{\infty}\right) \leq e^{\tau \int_{0}^{t} g^{q}(s) d s} g(t) \bar{\gamma}\left(\left\{x_{n}\right\}_{n=1}^{\infty}\right) .
\end{aligned}
$$

It is clear that $s \rightarrow(t-s)^{\alpha-1} g(s) \in L^{q}((0, t))$. Then, Lemma 6 implies

$$
\begin{aligned}
\chi\left(\left\{K\left(v_{n}\right)(t)\right\}_{n=1}^{\infty}\right) & \leq \bar{\gamma}\left(\left\{x_{n}\right\}_{n=1}^{\infty}\right) \frac{2}{\Gamma(\alpha)} \int_{0}^{t}(t-s)^{\alpha-1} g(s) e^{\tau} \int_{0}^{s} g^{q}(r) d r d s \\
& \leq \bar{\gamma}\left(\left\{x_{n}\right\}_{n=1}^{\infty}\right) \frac{2}{\Gamma(\alpha)}\left(\int_{0}^{t}(t-s)^{p \alpha-p} d s\right)^{\frac{1}{p}}\left(\int_{0}^{t} g^{q}(s) e^{q \tau} \int_{0}^{s} g^{q}(r) d r\right)^{\frac{1}{q}} d s \\
& \leq \bar{\gamma}\left(\left\{x_{n}\right\}_{n=1}^{\infty}\right) \frac{2 t^{\alpha-1+\frac{1}{p}}}{\sqrt[p]{(p \alpha-p+1)} \Gamma(\alpha)}\left(\int_{0}^{t} g^{q}(s) e^{q \tau \int_{0}^{s} g^{q}(r) d r}\right)^{\frac{1}{q}} d s \\
& \leq \bar{\gamma}\left(\left\{x_{n}\right\}_{n=1}^{\infty}\right) \frac{2 t^{\alpha-1+\frac{1}{p}}}{\sqrt[p]{q \tau(p \alpha-p+1)} \Gamma(\alpha)} e^{\tau \int_{0}^{t} g^{q}(s) d s} .
\end{aligned}
$$

Therefore,

$$
\bar{\gamma}\left(\left\{x_{n}\right\}_{n=1}^{\infty}\right) \leq \bar{\gamma}\left(\left\{h_{n}\right\}_{n=1}^{\infty}\right)=\sup _{t \in[0, b]} e^{-\tau \int_{0}^{t} g^{q}(s) d s} \chi\left(\left\{h_{n}(t)\right\}_{n=1}^{\infty}\right) \leq q_{*} \bar{\gamma}\left(\left\{x_{n}\right\}_{n=1}^{\infty}\right) .
$$

Using (19), we have

$$
\bar{\gamma}\left(\left\{x_{n}\right\}_{n=1}^{\infty}\right)=0 .
$$


Moreover, $\bar{\gamma}\left(x_{n}\right)=0$ implies that $\chi\left(\left\{x_{n}(t)\right\}\right)=0$ for a.e. $t \in[-r, T]$. In turn, (23) implies that

$$
\chi\left(\left\{v_{n}(t)\right\}\right)=0 \text {, for a.e. } t \in[0, T] .
$$

Hence, (21) implies that $\chi\left(\left\{h_{n}\right\}_{n=1}^{\infty}\right)=0$. By an argument similar to the one we used to prove that $N$ maps bounded sets into equicontinuous sets, we can prove that the set $\left\{h_{n}\right\}$ is equicontinuous, and so $\bmod _{C}(\mathrm{~B})=0$. It follows that $\gamma\left(\left\{h_{n}\right\}_{n=1}^{\infty}\right)=0$, which by (22) implies that $\gamma\left(\left\{x_{n}\right\}_{n=1}^{\infty}\right)=0$. Thus, $B$ is relatively compact. By the Arzelà-Ascoli theorem, $N$ is completely continuous, from which we have that $N$ is $\gamma$-condensing.

Step 4: $N$ has a closed graph. Let $\left\{x_{n}\right\}_{n \in \mathbb{N}} \in C([-r, T], E)$ be a sequence such that $\left\{x_{n}\right\}_{n \in \mathbb{N}}$ converges to $\widetilde{x}, h_{n} \in N\left(x_{n}\right)$, and $\left\{h_{n}\right\}_{n \in \mathbb{N}}$ converges to $\widetilde{h}$. We need to show that $\widetilde{h} \in N(\widetilde{x})$. So for each $n \in \mathbb{N}$ there exist $v_{n} \in S_{F}^{q}\left(x_{n}\right)$ such that

$$
h_{n}(t)=L\left(x_{n}(t)\right)+K\left(v_{n}(t)\right), \quad t \in J_{0} \cup J .
$$

Similar to the proof of Proposition 5, we can conclude that there exists a subsequence of $v_{n}$ converging weakly to $v$ in $L^{q}$ and satisfying

$$
v(t) \in F\left(t, \widetilde{x}_{t}\right), \quad \text { a.e. } t \in J .
$$

Since $K$ is a continuous linear operator,

$$
K\left(v_{n}(t)\right) \rightarrow K(v(t)) \text { as } n \rightarrow \infty .
$$

On the other hand, the continuity of $L$ implies that

$$
L\left(x_{n}(t)\right) \rightarrow L(\widetilde{x}(t)) \text { as } n \rightarrow \infty .
$$

Therefore,

$$
h_{n}(t) \rightarrow L(\widetilde{x}(t))+K(v(t)) \text { as } n \rightarrow \infty .
$$

This implies

$$
h=L(\widetilde{x}))+K(v) \in N(\widetilde{x}) .
$$

Hence, the multivalued operator $N$ has a closed graph.

In view of Steps 1-3, the proof of the proposition is complete.

We are now ready to give our main existence result for problem (12).

Theorem 7. Assume that $\left(\mathcal{H}_{1}\right)-\left(\mathcal{H}_{4}\right)$ hold. Then the problem (12) has at least one solution on $[-r, T]$ and the set FixN is compact.

Proof. It is clear that the solutions of Problem (12) correspond to the set FixN of fixed points of the multivalued operator $N$ defined in Proposition 6. By Propositions 5 and 6, $N(\cdot) \in \mathcal{P}_{c v, w c p}(C([-r, T], E))$ and it is u.s.c., admissible, and $\gamma$-condensing.

Let $x \in C([-r, T], E)$ be such that

$$
x \in \lambda N(x) \text { for some } 0<\lambda<1 .
$$

Then,

$$
\begin{aligned}
& \|x(t)\| \leq\|\varphi\|_{\infty}+\frac{1}{\Gamma(\alpha)} \int_{0}^{t}(t-s)^{\alpha-1} f(s) \psi\left(\left\|x_{s}\right\|\right) d s . \\
& \text { Let } \mu(t)=\sup _{-r \leq s \leq t}\|x(s)\| ; \text { then } \\
& \quad \mu(t) \leq\|\varphi\|_{\infty}+\frac{1}{\Gamma(\alpha)} \int_{0}^{t}(t-s)^{\alpha-1} f(s) \psi(\mu(s)) d s .
\end{aligned}
$$


From Corollary 3

$$
\mu(t) \leq \Psi^{-1}\left(\Psi(\bar{k})+\frac{t}{q}\right), t \in J
$$

where

$$
\Psi(z)=\int_{1}^{z} \frac{d x}{(\psi(x))^{q}}, \quad q\left(1-\frac{1}{p}\right)=1, \quad q>\frac{1}{\alpha}, \text { and } \bar{k}=\|\varphi\|_{\infty}+\frac{k^{p} T^{p(\alpha-1)+1}}{p(p(\alpha-1)+1)} .
$$

Hence,

$$
\|x\|_{\infty} \leq \Psi^{-1}\left(\Psi(\bar{k})+\frac{T}{q}\right)=: M_{*}
$$

and so the set

$$
\mathcal{M}=\{x \in C([-r, T], E): x \in \lambda N(x), \lambda \in(0,1)\}
$$

is bounded. As a consequence of Lemma $7, N$ has a fixed point $x$ in $U$ that is a solution to Problem (12). Finally, since FixN is bounded, by Lemma 8, FixN is also compact. This proves the theorem.

Remark 4. In all our results in Section 2 and in the main theorem in Section 5 (see condition $\left(\mathcal{H}_{3}\right)$, we have asked that $q>\frac{1}{\alpha}$. It would be of interest to see what might be proved in the case where $q \leq \frac{1}{\alpha}$.

\section{Conclusions}

We first established some new singular versions of the Gronwall-Bihari-Henry inequality. Then we proved a multivalued version of the Leray-Schauder alternative in strictly star-shaped sets. Using these new tools, we show how they can be applied to obtain new existence results for fractional differential inclusions with a delay.

Author Contributions: Conceptualization, Z.H., J.R.G., and A.O.; methodology, Z.H., J.R.G., and A.O.; formal analysis, Z.H., J.R.G., and A.O.; investigation, Z.H., J.R.G., and A.O.; writing-original draft preparation, Z.H., J.R.G., and A.O.; writing-review and editing, Z.H., J.R.G., and A.O.; visualization, Z.H., J.R.G., and A.O. All authors have read and agreed to the published version of the manuscript.

Funding: This research received no external funding.

Institutional Review Board Statement: Not applicable.

Informed Consent Statement: Not applicable.

Data Availability Statement: Not applicable.

Acknowledgments: The research of first and third authors has been partially supported by the General Direction of Scientific Research and Technological Development (DGRSDT), Algeria. The authors would like to thank the reviewers for their suggestion for improving the paper.

Conflicts of Interest: The authors declare no conflict of interest.

\section{References}

1. Bainov, D.; Simeonov, P. Integral Inequalities and Applications; Kluwer: Dordrecht, The Netherlands, 1992.

2. Bellman, R. Stability Theory of Differential Equations; McGraw-Hill: New York, NY, USA, 1953.

3. Henry, D. Geometric Theory of Semilinear Parabolic Partial Differential Equations; Springer: Berlin, Germany, 1989.

4. Lakshmikantham, V.; Leela, S. Differential and Integral Inequalities: Theory and Applications, Vol. II: Functional, Partial, Abstract, and Complex Differential Equations; Math. Science Engineering 55-II; Academic Press: New York, NY, USA, 1969.

5. Pachpatte, B.G. Inequalities for Differential and Integral Equations; Academic Press: New York, NY, USA, 1998.

6. Qin, Y. Analytic Inequalities and their Applications in PDEs; Operator Theory: Advances and Applications 241; Birkhäuser: Cham, Switzerland, 2017.

7. Qin, Y. Integral and Discrete Inequalities and Their Applications; Birkhäuser: Berlin, Germany, 2016.

8. Bihari, I. A generalization of a lemma of Bellman and its application to uniqueness problems of differential equations. Acta Math. Acad. Sci. Hungar. 1956, 7, 81-94. [CrossRef] 
9. Agarwal, R.P.; Deng, S.; Zhang, W. Generalization of a retarded Gronwall-like inequality and its applications. Appl. Math. Comput. 2005, 165, 599-612. [CrossRef]

10. Băleanu, D.; Machado, J.A.T.; Luo, A.C.J. Fractional Dynamics and Control; Springer: New York, NY, USA, 2012.

11. Gaul, L.; Klein, P.; Kempfle, S. Damping description involving fractional operators. Mech. Syst. Signal Process. 1991, 5, 81-88. [CrossRef]

12. Graef, J.R.; Kong, L.; Ledoan, A.; Wang, M. Modeling online social network dynamics using fractional-order epidemiological models. Math. Comput. Simul. 2020, 178, 625-645. [CrossRef]

13. Malinowska, A.B.; Torres, D.F.M. Towards a combined fractional mechanics and quantization. Fract. Calc. Appl. Anal. 2012, 15, 407-417. [CrossRef]

14. Metzler, F.; Schick, W.; Kilian, H.G.; Nonnenmacher, T.F. Relaxation in filled polymers: A fractional calculus approach. J. Chem. Phys. 1995, 103, 7180-7186. [CrossRef]

15. Nasholm, S.P.; Holm, S. On a fractional Zener elastic wave equation. Fract. Calc. Appl. Anal. 2013, 16, 26-50. [CrossRef]

16. Kilbas, A.A.; Srivastava, H.M.; Trujillo, J.J. Theory and Applications of Fractional Differential Equations; North-Holland Math. Studies, 204; Elsevier: Amsterdam, The Netherlands, 2006.

17. Podlubny, I. Fractional Differential Equations; Academic Press: San Diego, CA, USA, 1999.

18. Samko, S.G.; Kilbas, A.A.; Marichev, O.I. Fractional Integrals and Derivatives, Theory and Applications; Gordon and Breach: Yverdon, Switzerland, 1993.

19. Abdeljawad, T.; Alzabut, J. The $q$-fractional analogue for Gronwall-type inequality. J. Funct. Spaces Appl. 2013, 2013, 543839. [CrossRef]

20. Abdeljawad, T.; Alzabut, J.; Baleanu, D. A generalized $q$-fractional Gronwall inequality and its applications to nonlinear delay $q$-fractional difference systems. J. Inequal. Appl. 2016 2016, 240. [CrossRef]

21. Ding, X.L.; Cao-Labora, D.; Nieto, J.J. A new generalized Gronwall inequality with a double singularity and its applications to fractional stochastic differential equations. Stoch. Anal. Appl. 2019, 37, 1042-1056. [CrossRef]

22. Ferreira, R.A.C. A Discrete fractional Gronwall inequality. Proc. Am. Math. Soc. 2012, 140, 1605-1612. [CrossRef]

23. Ye, H.; Gao, J.; Ding, Y. A generalized Gronwall inequality and its application to a fractional differential equation. J. Math. Anal. Appl. 2007, 328, 1075-1081. [CrossRef]

24. Medved, M.A. A new approach to an analysis of Henry type integral inequalities and their Bihari type versions. J. Math. Anal. Appl. 1997, 214, 349-366. [CrossRef]

25. Medved, M. Integral inequalities and global solutions of semilinear evolution equations. J. Math. Anal. Appl. 2002, 267, 643-650. [CrossRef]

26. Dannan, F.M. Integral inequalities of Gronwall-Bellman-Bihari type and asymptotic behavior of certain second order nonlinear differential equations. J. Math. Anal. Appl. 1985, 108, 151-164. [CrossRef]

27. Aubin, J.P.; Frankowska, H. Set-Valued Analysis; Birkhaüser: Boston, MA, USA, 1990.

28. Graef, J.R.; Henderson, J.; Ouahab, A. Topological Methods for Differential Equations and Inclusions; Monographs and Research Notes in Mathematics Series; CRC Press: Boca Raton, FL, USA, 2019.

29. Deimling, K. Multi-Valued Differential Equations; De Gruyter: Berlin, Germany; New York, NY, USA, 1992.

30. Bothe, D. Multivalued perturbations of $m$-accretive differential inclusions. Israel J. Math. 1998, 108, 109-138. [CrossRef]

31. Pietkun, R. Structure of the solution set to Volterra integral inclusions and applications. J. Math. Anal. Appl. 2013, 403, 643-666. [CrossRef]

32. Górniewicz, L. Topological Fixed Point Theory of Multivalued Mappings; Springer: New York, NY, USA, 2006.

33. Akhmerov, R.R.; Kamenskij, M.I.; Potapov, A.S.; Rodkina, A.E.; Sadovskij, B.N. Measures of Noncompactness and Condensing Operators (in Russian) Nauka, Novosibirsk, 1986; Birkhäuser: Basel, Switzerland, 1992.

34. Kamenskii, M.; Obukhovskii, V.; Zecca, P. Condensing Multi-valued Maps and Semilinear Differential Inclusions in Banach Spaces; De Gruyter: Berlin, Germany, 2001.

35. Toledano, J.M.A.; Benavides, T.D.; Azedo, G.L. Measures of Noncompactness in Metric Fixed Point Theory; Birkhäuser: Basel, Switzerland, 1997.

36. Jiménez-Melado, A.; Morales, C.H. Fixed point theorems under the interior condition. Proc. Am. Math. Soc. 2006, 134, 501-507. [CrossRef]

37. González, C.; Jiménez-Melado, A.; Llorens-Fuster, E. A Mönch type fixed point theorem under the interior condition. J. Math Anal. Appl. 2009, 352, 816-821. [CrossRef]

38. Mönch, H. Boundary value problems for nonlinear ordinary differential equations of second order in Banach spaces. Nonlinear Anal. 1980, 4, 985-999. [CrossRef]

39. Pietkum, R. Solvability of inclusions of Hammerstein type. J. Math. Anal. Appl. 2019, 174, 351-380. [CrossRef]

40. Aubin, J.P.; Cellina, A. Differential Inclusions; Springer: Berlin/Heidelberg, Germany; New York, NY, USA, 1984.

41. Kisielewicz, M. Differential Inclusions and Optimal Control; Kluwer: Dordrecht, The Netherlands, 1991.

42. Filippov, A.F. Differential Equations with Discontinuous Right-hand Sides; Mathematics and its Applications (Soviet Series), 18; Kluwer: Dordrecht, The Netherlands, 1988.

43. Smirnov, G.V. Introduction to the Theory of Differential Inclusions; Graduate Studies Math. 41; Amer. Math. Soc.: Providence, RI, USA, 2002. 
44. Tolstonogov, A.A. Differential Inclusions in a Banach Space; Kluwer: Dordrecht, The Netherlands, 2000.

45. Andres, J.; Górniewicz, L. Topological Fixed Point Principles for Boundary Value Problems; Kluwer: Dordrecht, The Netherlands, 2003.

46. Djebali, S.; Górniewicz, L.; Ouahab, A. Solutions Sets for Differential Equations and Inclusions; De Gruyter Series in Nonlinear Analysis and Applications 18; De Gruyter: Berlin, Germany, 2013.

47. Hu, S.; Papageorgiou, N.S. Handbook of Multi-valued Analysis, Volume I: Theory; Kluwer: Dordrecht, The Netherlands, 1997.

48. Hu, S.; Papageorgiou, N.S. Handbook of Multi-valued Analysis. Volume II: Applications; Kluwer, Dordrecht, The Netherlands, 2000.

49. Abbas, S.; Benchohra, M.; Graef, J.R.; Henderson, J. Implicit Fractional Differential and Integral Equations; De Gruyter Series in Nonlinear Analysis and Applications; De Gruyter: Berlin, Germany, 2018; Volume 26.

50. Abbas, S.; Benchohra, M.; N'Gurékata, G.N. Advanced Fractional Differential and Integral Equations; Nova Science Publishers: New York, NY, USA, 2014.

51. Benchohra, M.; Henderson, J.; Ntouyas, S.K.; Ouahab, A. Existence results for fractional order functional differential equations with infinite delay. J. Math. Anal. Appl. 2008, 338, 1340-1350. [CrossRef]

52. Benchohra, M.; Henderson, J.; Ntouyas, S.K.; Ouahab, A. Existence results for fractional functional differential inclusions with infinite delay and application to control theory. Fract. Calc. Appl. Anal. 2008, 11, 35-56.

53. Ravichandran, C.; Valliammal, N.; Nieto, J.J. New results on exact controllability of a class of fractional neutral integro-differential systems with state-dependent delay in Banach spaces. J. Franklin Inst. 2019, 356, 1535-1565. [CrossRef]

54. Valliammal, N.; Ravichandran, C.; Nisar, K.S. Solutions to fractional neutral delay differential nonlocal system. Chaos Solitons Fractals 2020, 138, 109912. [CrossRef]

55. Zhou, Y.; Wang, J.; Zhang, L. Basic Theory of Fractional Differential Equations, 2nd ed.; World Scientific: Hackensack, NJ, USA, 2017.

56. Diestel, J.; Uhl, J.J., Jr. Vector Measures; Math. Surveys 15; American Mathematical Society: Providence, RI, USA, 1977.

57. Brezis, H. Functional Analysis, Sobolev Spaces and Partial Differential Equations; Springer: New York, NY, USA, 2011. 